\title{
Quantum-classical correspondence via Liouville dynamics. I. Integrable systems and the chaotic spectral decomposition
}

\author{
Joshua Wilkie and Paul Brumer \\ Chemical Physics Theory Group, Department of Chemistry, University of Toronto, Toronto, Ontario, Canada M5S 3 H6
}

(Received 14 June 1996)

\begin{abstract}
A general program to show quantum-classical correspondence for bound conservative integrable and chaotic systems is described. The method is applied to integrable systems and the nature of the approach to the classical limit, the cancellation of essential singularities, is demonstrated. The application to chaotic systems requires an understanding of classical Liouville eigenfunctions and a Liouville spectral decomposition, developed herein. General approaches to the construction of these Liouville eigenfunctions and classical spectral projectors in quantum and classical mechanics are discussed and are employed to construct Liouville eigenfunctions for classically chaotic systems. Correspondence for systems whose classical analogs are chaotic is discussed, based on this decomposition, in the following paper [Phys. Rev. A 54, 43 (1996)].
\end{abstract}

[S1050-2947(96)05112-8]

PACS number(s): 03.65.-w

\section{INTRODUCTION}

The correspondence principle requires that the laws of quantum dynamics reproduce the predictions of classical mechanics when the energy and mass of a dynamical system are very large with respect to Planck's constant [1]. In other words, the correspondence principle asserts that the fundamental laws of physics that are valid on the atomic scale are also responsible for the observed classical dynamics of macroscopic systems. It is therefore surprising, considering the important conceptual role of the correspondence principle, that there is little direct evidence to support its general validity [2-4]. In fact it has been controversially [4,5] argued that the absence of chaos in quantum dynamics plus its presence in the dynamics of classical systems prevents any possibility of correspondence [3]. The main goal of this paper and its companion [6] is to provide a consistent theory of correspondence in both integrable and chaotic systems.

To understand correspondence requires that we first adopt a framework in which quantum and classical dynamics can be realistically compared. The Liouville picture in classical mechanics in combination with a phase-space representation of quantum dynamics provides such a framework. Here both classical and quantum mechanics are represented by objects defined on phase space and by operators defined on these objects [7]. Our correspondence approach is then based on the desire to prove that the quantum dynamics of phasespace distributions obeys classical mechanics in the classical limit. We do so by focusing on the correspondence of what we argue to be the essential elements in dynamics, the eigendistributions and eigenvalues of the classical and quantum Liouville operators [8,9] (the Poisson bracket with the classical Hamiltonian $H$, and the commutator with the Hamiltonian operator $\hat{H}$, respectively).

We outline this correspondence program in Sec. II and describe the progress made and developments required in the application to regular and chaotic systems. The approach emphasizes the significance of the eigenfunctions of the classical Liouville operator and of the classical spectral projectors.
These eigenfunctions are obtained in Sec. III for both regular and chaotic systems. The correspondence program is then implemented for integrable systems in Sec. IV and the nonsingular nature of the approach to the classical limit is displayed. This approach to correspondence proves inappropriate for chaotic systems. In this case a description of the dynamics in terms of classical spectral projectors is required. These quantities are introduced and discussed in Sec. V. A companion paper [6] shows quantum-classical correspondence for chaotic systems.

\section{LIOUVILLE PICTURE: THE CORRESPONDENCE PROGRAM}

Arguments in favor of the Liouville picture as the conceptual framework most suited for the study of correspondence have been presented elsewhere $[10,11]$. Here we briefly summarize. First, the Liouville picture of classical dynamics, which deals with the time evolution of phase-space densities, is conceptually superior to the trajectory viewpoint because it implicitly recognizes the imperfect nature of classical preparation and measuring devices. That is, one cannot initially prepare the exact points in phase space that define a single trajectory. Similarly, the limitations of quantum mechanical preparation and measurement can be incorporated in the von Neumann (quantum Liouville) equation for the density matrix; i.e., the von Neumann equation admits solutions that are not states of maximal information. Thus, the Liouville equation plays a similar role in quantum and classical dynamics. Second, our approach takes advantage of the similarity between the dynamics of classical distributions in phase space, as governed by the classical Liouville equation, and the dynamics of quantum density matrices, governed by the von Neumann equation in the Wigner-Weyl representation. Adopting this perspective, both classical and quantum mechanics have similar structures and rely upon similar quantities. Finally, formal classifications of classical systems as integrable, ergodic, mixing, etc. are most properly done in terms of the eigenfunctions and eigenvalues of the classical Liouville operator [12], i.e., the Poisson bracket with the 
Hamiltonian. Hence this provides the most fundamental of frameworks.

The time evolution of the quantum density matrix $\hat{\rho}$ is given by the solution to the von Neumann (or quantum Liouville) equation:

$$
\frac{\partial \hat{\rho}}{\partial t}=-i \hbar^{-1}[\hat{H}, \hat{\rho}]=-i L \hat{\rho}
$$

where $L=\hbar^{-1}[\hat{H}$,$] is the quantum Liouville operator, and$ $[$,$] is the commutator. Solutions to Eq. (1) are of the form$

$$
\hat{\rho}=\hat{\rho}_{\lambda, \alpha} e^{-i \lambda t}
$$

if $\hat{\rho}_{\lambda, \alpha}$ satisfies the eigenvalue problem

$$
L \hat{\rho}_{\lambda, \alpha}=\lambda \hat{\rho}_{\lambda, \alpha}
$$

The label $\alpha$ is introduced to accommodate degeneracies associated with the eigenvalue $\lambda$. In particular, a complete characterization of the quantum Liouville eigenvalue problem requires the specification of a complete set of commuting superoperators in the same way that solution of the Schrödinger eigenvalue problem requires a complete set of commuting observables [8]. This complete set of commuting superoperators can be constructed in the following way. Suppose

$$
\hat{H}, \hat{K}_{1}, \ldots, \hat{K}_{r^{\prime}-1}
$$

for $r^{\prime} \leqslant s$ is a complete set of observables for a system of $s$ degrees of freedom such that $\left[\hat{H}, \hat{K}_{i}\right]=0$ and $\left[\hat{K}_{i}, \hat{K}_{j}\right]=0$ for all $i, j \leqslant r^{\prime}-1$. It follows that the complete set of $2 r^{\prime}$ commuting quantum superoperators is

$$
L, \mathcal{H}, \frac{1}{\hbar}\left[\hat{K}_{1},\right], \frac{1}{2}\left[\hat{K}_{1},\right]_{+}, \ldots, \frac{1}{\hbar}\left[\hat{K}_{r^{\prime}-1},\right], \frac{1}{2}\left[\hat{K}_{r^{\prime}-1},\right]_{+},
$$

where $\mathcal{H}=\frac{1}{2}[\hat{H},]_{+}$is the energy superoperator, and $[,]_{+}$ denotes the anticommutator, i.e., $[\hat{A}, \hat{B}]_{+}=\hat{A} \hat{B}+\hat{B} \hat{A}$. A Liouville eigenstate for the system is completely specified if it is an eigenstate of all $2 r^{\prime}$ superoperators. Thus $\hat{\rho}_{\lambda, \alpha}$ in Eq. (3) is an eigenfunction of the collection of superoperators in Eq. (5), with the label $\alpha$ denoting the eigenvalues of all superoperators other than $L$.

Equations (1) and (3) are of the following form in the Wigner-Weyl representation $[13][\mathbf{x}=(\mathbf{p}, \mathbf{q})$ denotes the collection of momenta $\mathbf{p}$ and coordinates $\mathbf{q}]$

$$
\frac{\partial \rho^{w}(\mathbf{x}, t)}{\partial t}=-i L(\mathbf{x}) \rho^{w}(\mathbf{x}, t)
$$

and

$$
L(\mathbf{x}) \rho_{\lambda, \alpha}^{w}(\mathbf{x})=\lambda \rho_{\lambda, \alpha}^{w}(\mathbf{x})
$$

where

$$
\begin{aligned}
L(\mathbf{x}) & =\frac{2}{\hbar} i H(\mathbf{x}) \sin (\hbar \sigma / 2) \\
& =\frac{2}{\hbar} i H(\mathbf{x}) \sin \left[\frac{\hbar}{2}\left(\frac{\overleftarrow{\partial}}{\partial \mathbf{q}} \cdot \frac{\overleftrightarrow{\partial}}{\partial \mathbf{p}}-\frac{\overleftarrow{\partial}}{\partial \mathbf{p}} \cdot \frac{\vec{\partial}}{\partial \mathbf{q}}\right)\right] .
\end{aligned}
$$

Here $\sigma$ implies the Poisson bracket, i.e.,

$$
A(\mathbf{x}) \sigma B(\mathbf{x})=\{A, B\}=\frac{\partial A(\mathbf{x})}{\partial \mathbf{q}} \cdot \frac{\partial B(\mathbf{x})}{\partial \mathbf{p}}-\frac{\partial A(\mathbf{x})}{\partial \mathbf{p}} \cdot \frac{\partial B(\mathbf{x})}{\partial \mathbf{q}}
$$

and the arrows over the derivatives indicate that the derivative is taken of the function preceding or following the derivative operator.

Given the solution to Eq. (7), the dynamics of any distribution $\rho^{w}(\mathbf{x}, t)$, with initial condition $\rho^{w}(\mathbf{x}, t=0)=\rho(\mathbf{x}, 0)$, can be written as

$$
\rho^{w}(\mathbf{x}, t)=\sum_{\lambda, \alpha} c_{\lambda, \alpha} \rho_{\lambda, \alpha}^{w}(\mathbf{x}) e^{-i \lambda t}
$$

where

$$
c_{\lambda, \alpha}=\int d \mathbf{x}_{0} \rho\left(\mathbf{x}_{0}, 0\right) \rho_{\lambda, \alpha}^{w *}\left(\mathbf{x}_{0}\right)
$$

That is,

$$
\rho^{w}(\mathbf{x}, t)=\int d \mathbf{x}_{0} \rho\left(\mathbf{x}_{0}, 0\right)\left[\sum_{\lambda, \alpha} \rho_{\lambda, \alpha}^{w}(\mathbf{x}) \rho_{\lambda, \alpha}^{w *}\left(\mathbf{x}_{0}\right)\right] e^{-i \lambda t} .
$$

Consider now the analogous approach in classical mechanics, which considers the dynamics of phase-space distributions $\rho(\mathbf{x}, t)$. In particular, $\rho(\mathbf{x}, t)$ satisfies the Liouville equation

$$
\frac{\partial \rho(\mathbf{x}, t)}{\partial t}=-i L_{c} \rho(\mathbf{x}, t)
$$

where the classical Liouville operator $L_{c}$ is given by

$$
L_{c}=i\{H(\mathbf{x}),\} \text {. }
$$

Here $H(\mathbf{x})$ is the Hamiltonian and $\{$,$\} denotes the Poisson$ bracket [Eq. (9)]. The time evolution of $\rho(\mathbf{x}, t)$ is given by

$$
\rho(\mathbf{x}, t)=\rho_{\lambda^{c}, \alpha}(\mathbf{x}) e^{-i \lambda^{c} t}
$$

if $\rho_{\lambda^{c}, \alpha}(\mathbf{x})$ satisfies the eigenvalue problem

$$
L_{c} \rho_{\lambda^{c}, \alpha}(\mathbf{x})=\lambda^{c} \rho_{\lambda^{c}, \alpha}(\mathbf{x}) .
$$

Once again $\alpha$ contains the labeling associated with states that are $\lambda^{c}$ degenerate. In particular, a complete characterization of the classical Liouville eigenvalue problem requires the specification of a complete set of commuting operators on the classical Hilbert space $[11,14]$. Suppose the classical ana$\log$ of our quantum system has $r$ independent constants of motion. Then we can construct $r-1$ functions $K_{j}(\mathbf{x})$, which are constants of the motion, i.e., $\left\{H(\mathbf{x}), K_{j}(\mathbf{x})\right\}=0$, and 
which are in involution so that $\left\{K_{i}(\mathbf{x}), K_{j}(\mathbf{x})\right\}=0$ for all $i, j \leqslant r-1$. The complete set of commuting operators on the classical Hilbert space is then

$$
L_{c}, H(\mathbf{x}), i\left\{K_{1}(\mathbf{x}),\right\}, K_{1}(\mathbf{x}), \ldots, i\left\{K_{r-1}(\mathbf{x}),\right\}, K_{r-1}(\mathbf{x}),
$$

where $L_{c}, i\left\{K_{1}(\mathbf{x}),\right\}, \ldots, i\left\{K_{r-1}(\mathbf{x}),\right\}$ are first-order linear differential operators and $H(\mathbf{x}), K_{1}(\mathbf{x}), \ldots, K_{r-1}(\mathbf{x})$ are multiplicative operators. A Liouville eigenstate for the classical problem is usually completely specified if it is an eigenstate of all $2 r$ classical operators [14].

Given such solutions, the evolution of any classical distribution $\rho(\mathbf{x}, t)$ can be written as

$$
\rho(\mathbf{x}, t)=\sum_{\lambda^{c}, \alpha} c_{\lambda^{c}, \alpha} \rho_{\lambda^{c}, \alpha}(\mathbf{x}) e^{-i \lambda^{c} t},
$$

where

$$
c_{\lambda^{c}, \alpha}=\int d \mathbf{x}_{0} \rho\left(\mathbf{x}_{0}, 0\right) \rho^{*}{ }_{\lambda^{c}, \alpha}\left(\mathbf{x}_{0}\right)
$$

or

$$
\rho(\mathbf{x}, t)=\int d \mathbf{x}_{0} \rho\left(\mathbf{x}_{0}, 0\right)\left[\sum_{\lambda^{c}, \alpha} \rho_{\lambda^{c}, \alpha}(\mathbf{x}) \rho^{*}{ }_{\lambda^{c}, \alpha}\left(\mathbf{x}_{0}\right)\right] e^{-i \lambda^{c} t} .
$$

Both Eqs. (12) and (20) express the time evolution of the phase-space density in terms of the initial distribution $\rho\left(\mathbf{x}_{0}, 0\right)$, the eigenvalues $\lambda, \lambda^{c}$, and a term comprised of products of Liouville eigenfunctions contained within brackets. This rewriting of the right-hand sides of the equations, and the term in brackets in particular, will prove to play a central role in the correspondence program we develop in these papers. Indeed the term in brackets, in Eqs. (12) and (20), is related to the Liouville spectral projection operators, which are heavily emphasized in this paper.

A comparison of Eqs. (12) and (20) motivates our correspondence approach. Specifically, it suggests that one can show quantum-classical correspondence by showing that the $h \rightarrow 0$ limit of the quantum Liouville eigenfunctions and its eigenvalues are the classical Liouville eigenfunctions and eigenvalues. This approach has proven successful for integrable systems (see Ref. [10] and Sec. IV B below). However, as shown below and in the following paper [6], it is an incorrect approach for chaotic systems. In such cases the quantum Liouville eigenstates have no classical limit. Rather, one must deal with quantum and classical spectral projection operators, which have singularity-free classical limits. These are related to the objects contained within brackets in Eqs. (12) and (20).

The status of this correspondence program is readily summarized for integrable systems $\left(r=r^{\prime}=s\right)$. Berry [15] demonstrated correspondence for the stationary Liouville eigenfunctions $|\mathbf{n}\rangle\langle\mathbf{n}|$ where $|\mathbf{n}\rangle$ are eigenstates of the Hamiltonian operator with corresponding energies $E_{\mathbf{n}}$ and the $s$ integers $\mathbf{n}$ label the quantum states of the Schrodinger picture. Specifically, he showed that

$$
\begin{aligned}
\rho_{\mathbf{n}, \mathbf{n}}^{w}(\mathbf{x}) & \equiv h^{-s / 2} \int d \mathbf{v} e^{i \mathbf{p} \cdot \mathbf{v} / \hbar}\langle\mathbf{q}-\mathbf{v} / 2 \mid \mathbf{n}\rangle\langle\mathbf{n} \mid \mathbf{q}+\mathbf{v} / 2\rangle \\
& \rightarrow \hbar^{s / 2} \rho_{\mathbf{I}_{\mathbf{n}}, \mathbf{0}}(\mathbf{x})
\end{aligned}
$$

in the $h \rightarrow 0$ limit, where $\mathbf{I}_{\mathbf{n}}=(\mathbf{n}+\boldsymbol{\beta}) \hbar$, the constants $\boldsymbol{\beta}$ are the Maslov indices $[10,16], \rho_{\mathbf{n}, \mathbf{n}}^{w}$ is the Wigner transform of the stationary Liouville eigenstate $|\mathbf{n}\rangle\langle\mathbf{n}|$, and the classical Liouville eigenfunctions $\rho_{\mathbf{I}^{\prime}, \mathbf{k}}(\mathbf{x})$ are defined below [Eq. (30)]. Correspondence for the nonstationary Liouville eigenfunctions $|\mathbf{n}\rangle\langle\mathbf{m}|$ and eigenvalues $\lambda_{\mathbf{n}, \mathbf{m}}=\left(E_{\mathbf{n}}-E_{\mathbf{m}}\right) / \hbar$ was considered by Jaffé and Brumer [10]. In particular, they examined the Wigner transform $\rho_{\mathbf{n}, \mathbf{m}}^{w}(\mathbf{x})$ of $|\mathbf{n}\rangle\langle\mathbf{m}|$ and showed that for $\mathbf{n} \neq \mathbf{m}$,

$$
\begin{aligned}
\rho_{\mathbf{n}, \mathbf{m}}^{w}(\mathbf{x}) & \equiv h^{-s / 2} \int d \mathbf{v} e^{i \mathbf{p} \cdot \mathbf{v} / \hbar}\langle\mathbf{q}-\mathbf{v} / 2 \mid \mathbf{n}\rangle\langle\mathbf{m} \mid \mathbf{q}+\mathbf{v} / 2\rangle \\
& \rightarrow \hbar^{s / 2} \rho_{\mathbf{I}_{\mathbf{n}, \mathbf{m}}, \mathbf{n}-\mathbf{m}}(\mathbf{x}),
\end{aligned}
$$

and

$$
\lambda_{\mathbf{n}, \mathbf{m}} \rightarrow \lambda_{\mathbf{I}_{\mathbf{n}, \mathbf{m}}, \mathbf{n}-\mathbf{m}}^{c}
$$

as $h \rightarrow 0$, where they surmised that $\mathbf{I}_{\mathbf{n}, \mathbf{m}}=\left(\mathbf{I}_{\mathbf{n}}+\mathbf{I}_{\mathbf{m}}\right) / 2$. However, as we will later show, this $\mathbf{I}_{\mathbf{n}, \mathbf{m}}$ is an approximation to the exact result, which depends intimately on the eigenvalues of the complete set of commuting observables [Eq. (4)]. These classical Liouville eigenvalues $\lambda_{\mathbf{I}^{\prime}, \mathbf{k}}^{c}$ are defined below [Eq. (31)].

By contrast, the correspondence program for systems whose classical analogs are chaotic has been left largely undeveloped. The nature of the classical Liouville eigenfunctions and eigenspectrum is not clearly understood [17], and only the correspondence limits of the stationary Liouville eigenfunctions have been explored. Berry [18] and Voros [19] were able to show that the Wigner function associated with a stationary Liouville eigenstate $|n\rangle\langle n|$ (here $\left.r=r^{\prime}=1\right)$ concentrates in the classical limit to a uniform distribution over the classical energy surface of the same energy $E_{n}$, i.e.,

$$
\rho_{n, n}^{w}(\mathbf{x}) \rightarrow h^{s / 2} \frac{\delta\left(E_{n}-H(\mathbf{x})\right)}{\int d \mathbf{x}^{\prime} \delta\left(E_{n}-H\left(\mathbf{x}^{\prime}\right)\right)} .
$$

Investigations of the semiclassical corrections to this limit have shown that the unstable periodic orbits of the chaotic classical system strongly influence the quantum eigenfunctions, resulting in accentuations of probability in the vicinity of periodic orbits $[4,20]$. Semiclassical corrections have also been studied for the stationary Liouville eigenfunctions of regular systems [15]. However, no results have been obtained for the correspondence of the nonstationary Liouville eigenfunctions or eigenspectrum for the chaotic case. The reason for this is made clear in the following paper, where we show that the $\rho_{n, m}^{w}(\mathbf{x}), n \neq m$, for chaotic systems do not have classical limits. Rather, one must deal with the classical and quantum spectral projection operators discussed below. 


\section{CLASSICAL LIOUVILLE EIGENFUNCTIONS AND EIGENSPECTRUM}

The classical Liouville eigenvalue problem [Eq. (16)] requires that one find a complete set of orthogonal Liouville eigenfunctions $\rho_{\lambda, \alpha}(\mathbf{x})$ with eigenvalues $\lambda$; i.e., they must satisfy

$$
\int d \mathbf{x} \rho_{\lambda^{\prime}, \alpha^{\prime}}^{*}(\mathbf{x}) \rho_{\lambda, \alpha}(\mathbf{x})=0
$$

for $\lambda \neq \lambda^{\prime}$ or $\alpha \neq \alpha^{\prime}$, and

$$
\sum_{\lambda, \alpha} \rho_{\lambda, \alpha}^{*}\left(\mathbf{x}_{0}\right) \rho_{\lambda, \alpha}(\mathbf{x})=\delta\left(\mathbf{x}-\mathbf{x}_{0}\right)
$$

(Note that for notational simplicity, classical eigenvalues in the remainder of the paper are denoted $\lambda$, rather than $\lambda^{c}$.) For the case of $s$ degrees of freedom and $r$ constants of the motion we must find simultaneous eigenstates of the complete set of $2 r$ operators [Eq. (17)].

If a distribution $f(\mathbf{x})$ is an eigenstate of a real multiplicative operator $A(\mathbf{x})$ with eigenvalue $A^{\prime}$, then $\left[A(\mathbf{x})-A^{\prime}\right] f(\mathbf{x})=0$. That is, $f(\mathbf{x})=0$ for all $\mathbf{x}$ not satisfying $A(\mathbf{x})=A^{\prime}$ so that all nontrivial solutions $f(\mathbf{x})$ must be proportional to $\delta\left(A(\mathbf{x})-A^{\prime}\right)$. That is, all distributions that are eigenstates of the multiplicative operators $H(\mathbf{x}), K_{1}(\mathbf{x}), \ldots, K_{r-1}(\mathbf{x})$ must be proportional to

$$
\delta(E-H(\mathbf{x})) \Pi_{j=1}^{r-1} \delta\left(K_{j}(\mathbf{x})-K_{j}^{\prime}\right)
$$

Little more can be said about the general case of arbitrary $r$. We therefore focus on the two interesting special cases: $r=s$, corresponding to integrable systems, and $r=1$, which includes the case of chaotic motion.

\section{A. Integrable systems}

For $r=s$ we can introduce action variables $\mathbf{I}$ and conjugate angle variables $\theta$ such that $H(\mathbf{x})=H(\mathbf{I})$, and $K_{j}(\mathbf{x})=K_{j}(\mathbf{I})$ for $j=1, \ldots, s-1$. It follows that there exists an $\mathbf{I}^{\prime}$ such that

$$
\delta(E-H(\mathbf{x})) \Pi_{j=1}^{r-1} \delta\left(K_{j}(\mathbf{x})-K_{j}^{\prime}\right) \propto \delta\left(\mathbf{I}(\mathbf{x})-\mathbf{I}^{\prime}\right)
$$

and that the classical Liouville eigendistributions $\rho_{\lambda, \alpha}(\mathbf{x})$ must be of the form $\delta\left(\mathbf{I}(\mathbf{x})-\mathbf{I}^{\prime}\right) F(\boldsymbol{\theta}(\mathbf{x}))$. Since $L_{c}=-i \boldsymbol{\omega}(\mathbf{I}) \cdot \partial / \partial \boldsymbol{\theta}$ the Liouville eigenequation is

$$
-i \boldsymbol{\omega}\left(\mathbf{I}^{\prime}\right) \cdot \frac{\partial}{\partial \boldsymbol{\theta}} F(\boldsymbol{\theta})=\lambda F(\boldsymbol{\theta})
$$

with solutions $F(\boldsymbol{\theta})=e^{i \mathbf{a} \cdot \boldsymbol{\theta}}, \lambda=\mathbf{a} \cdot \boldsymbol{\omega}\left(\mathbf{I}^{\prime}\right)$. If the system is periodic in the angle variables then $\mathbf{a}=\mathbf{k} \in \mathbf{Z}^{s}$, i.e., $\mathbf{a}$ is a vector of $s$ integers. Thus the classical Liouville eigenfunctions for an integrable system are given by

$$
\rho_{\mathbf{I}^{\prime}, \mathbf{k}}(\mathbf{x})=\frac{1}{(2 \pi)^{s / 2}} \delta\left(\mathbf{I}^{\prime}-\mathbf{I}\right) e^{i \mathbf{k} \cdot \boldsymbol{\theta}}
$$

with eigenvalue

$$
\lambda_{\mathbf{I}^{\prime}, \mathbf{k}}=\mathbf{k} \cdot \boldsymbol{\omega}\left(\mathbf{I}^{\prime}\right)
$$

Note that these eigenfunctions are also eigenfunctions of the differential operators $i\left\{K_{j}(\mathbf{x}),\right\}=-i \partial K_{j}(\mathbf{I}) / \partial \mathbf{I} \cdot \partial / \partial \boldsymbol{\theta}$ with corresponding eigenvalues $\mathbf{k} \cdot \partial K_{j}\left(\mathbf{I}^{\prime}\right) / \partial \mathbf{I}^{\prime}$. If the $K_{j}(\mathbf{I})=I_{j}$ then the eigenvalues are simply $k_{j}$. This set of eigenfunctions is complete and orthogonal, i.e.,

$$
\sum_{\mathbf{k}} \int d \mathbf{I}^{\prime} \rho_{\mathbf{I}^{\prime}, \mathbf{k}}^{*}\left(\mathbf{x}^{\prime}\right) \rho_{\mathbf{I}^{\prime}, \mathbf{k}}(\mathbf{x})=\delta\left(\mathbf{x}-\mathbf{x}^{\prime}\right),
$$

and

$$
\int d \mathbf{x} \rho_{\mathbf{I}^{\prime \prime}, \mathbf{k}^{\prime}}^{*}(\mathbf{x}) \rho_{\mathbf{I}^{\prime}, \mathbf{k}}(\mathbf{x})=\delta_{\mathbf{k}, \mathbf{k}^{\prime}} \delta\left(\mathbf{I}^{\prime}-\mathbf{I}^{\prime \prime}\right)
$$

We comment briefly on the character of the Liouville eigenfunctions for integrable systems; a detailed discussion and related computations are provided elsewhere [11]. The spectrum of an integrable system on a given torus $\mathbf{I}^{\prime}=\mathbf{I}(\mathbf{x})$ is discrete and is given by the set of frequencies $\left\{\mathbf{k} \cdot \boldsymbol{\omega}\left(\mathbf{I}^{\prime}\right) \mid \mathbf{k}\right.$ $\left.\in \mathbf{Z}^{s}\right\}$. On the energy surface $E=H(\mathbf{x})$, the spectrum of an integrable system is the union of all sets $\left\{\mathbf{k} \cdot \boldsymbol{\omega}\left(\mathbf{I}^{\prime}\right) \mid \mathbf{k} \in \mathbf{Z}^{s}\right\}$ from tori $\mathbf{I}^{\prime}=\mathbf{I}(\mathbf{x})$ with $H\left(\mathbf{I}^{\prime}\right)=E$. Typically, $\boldsymbol{\omega}\left(\mathbf{I}^{\prime}\right)$ varies with $\mathbf{I}^{\prime}$, so that the spectrum of an integrable system on an energy surface is continuous. In our terminology [21] eigenfunctions with frequencies $\lambda_{\mathbf{I}^{\prime}, \mathbf{k}}=\mathbf{k} \cdot \boldsymbol{\omega}\left(\mathbf{I}^{\prime}\right)$ with $\mathbf{k} \neq 0$ belong to the continuous spectrum while those with $\mathbf{k}=0$ belong to the point spectrum. Examination of Eq. (30) reveals that the eigenfunctions $\rho_{\mathbf{I}^{\prime}, \mathbf{k}}(\mathbf{I}, \boldsymbol{\theta})$ are identically zero off the torus $\mathbf{I}(\mathbf{x})=\mathbf{I}^{\prime}$. Eigendistributions with $\mathbf{k}=0$ are stationary and uniform on the torus, and as a set they project out the longtime limit of any given initial probability distribution. For $\mathbf{k} \neq 0$ the character of the eigendistribution is determined by the underlying orbit structure of the torus $\mathbf{I}(\mathbf{x})=\mathbf{I}^{\prime}$. If the frequencies $\omega_{1}, \omega_{2}, \ldots, \omega_{s}$ of the torus are commensurate then the orbits of the torus $\mathbf{I}(\mathbf{x})=\mathbf{I}^{\prime}$ are all periodic [22]. The eigendistributions associated with commensurate tori are nonuniform and stationary when $\mathbf{k} \cdot \boldsymbol{\omega}\left(\mathbf{I}^{\prime}\right)=0$ and $\mathbf{k} \neq 0$, and nonuniform and nonstationary when $\mathbf{k} \cdot \boldsymbol{\omega}\left(\mathbf{I}^{\prime}\right) \neq 0$.

The eigendistributions associated with incommensurate tori are nonuniform and nonstationary for $\mathbf{k} \neq 0$. Eigendistributions for integrable systems are supported only over a given torus $\mathbf{I}^{\prime}=\mathbf{I}(\mathbf{x})$, but every orbit of the torus contributes to the construction of the eigenfunction [23].

\section{B. Chaotic systems}

Now consider the case where $r=1$. In addition to being chaotic we assume that the system is hyperbolic, i.e., that it has a countable number of isolated unstable periodic orbits on each surface of constant energy. Thus, our chaotic system has positive Kolmogorov entropy, exhibits sensitivity to initial conditions (i.e., possesses $2 s-2$ nonvanishing Liapunov exponents), and is mixing, and hence is weak mixing and ergodic [12]. Such familiar properties of chaotic systems (i.e., ergodicity, weak mixing, mixing, etc.) may be related to the spectral properties of the Liouville operator [12]. For example, if $\lambda=0$ is a nondegenerate point eigenvalue of the Liouville operator $L_{c}$ on the energy surface then the system is ergodic. If $\lambda=0$ is the only point eigenvalue of $L_{c}$ on the energy surface then the system is weak mixing. If the rest of the spectrum is continuous then the system is mixing. In this 
section we determine the form of the generalized eigenfunctions that correspond to the point and continuous Liouville eigenspectrum [12,21] for chaotic systems.

In accord with Eq. (17) we seek eigenfunctions of the complete set $L_{c}$ and $H(\mathbf{x})$. As eigenstates of the energy multiplication operator $H(\mathbf{x})$ they must be proportional to $\delta(E-H(\mathbf{x}))$ where $E$ is the corresponding energy eigenvalue. Consider then the function $\tau(\mathbf{x})$, which is conjugate to the constant of the motion $H(\mathbf{x})$, i.e., $\{\tau(\mathbf{x}), H(\mathbf{x})\}=1$. Then the distribution $\delta(E-H(\mathbf{x})) e^{i \lambda \tau(\mathbf{x})}$ is an eigenfunction of the classical Liouville operator with eigenvalue $\lambda$ since $L_{c}=i\{H(\mathbf{x})\}=,-i \partial / \partial \tau$. Note that $e^{-i L_{c} t} \tau(\mathbf{x})=\tau(\mathbf{X}(\mathbf{x}$, $-t))=\tau(\mathbf{x})-t$, where $\mathbf{X}(\mathbf{x},-t)$ is the phase-space point from which $\mathbf{x}$ evolves over the time $t$. Thus, for a given choice of the origin of time along each orbit of the system, we can construct a function $\tau(\mathbf{x})$, which specifies the time of an arbitrary point $\mathbf{x}$ along the orbit, since trajectories do not intersect one another in phase space. However, the distributions $\delta(E-H(\mathbf{x})) e^{i \lambda \tau(\mathbf{x})}$ [24] do not form a complete set of Liouville eigenfunctions since they do not account for the infinite degeneracy of the classical Liouville spectrum for chaotic systems [12].

To properly define eigenfunctions we must consider the manner in which points in phase space are labeled. Specifically we introduce a system of variables: $H(\mathbf{x})$, which is the energy of a point in phase space, $\tau(\mathbf{x})$ a function that assigns a time to every point in phase space, and $2 s-2$ stationary variables $\eta(\mathbf{x})$, which label the trajectories on the energy surface. The functions $\tau$ and $\boldsymbol{\eta}$ are continuous only along an orbit: $\tau$ increments smoothly while $\eta$ remains constant along an orbit. The $\boldsymbol{\eta}$ variables are chosen to have the property that

$$
\begin{aligned}
\delta\left(\mathbf{x}_{0}-\mathbf{x}\right)= & \delta\left(H\left(\mathbf{x}_{0}\right)-H(\mathbf{x})\right) \delta\left(\tau\left(\mathbf{x}_{0}\right)\right. \\
& -\tau(\mathbf{x})) \delta\left(\boldsymbol{\eta}\left(\mathbf{x}_{0}\right)-\boldsymbol{\eta}(\mathbf{x})\right) .
\end{aligned}
$$

As one moves off a trajectory the variables $\tau$ and $\boldsymbol{\eta}$ must take on all possible values infinitely often since (a) there are an infinite number of chaotic trajectories, (b) every chaotic trajectory comes arbitrarily close to every phase space point on every other trajectory, and (c) chaotic trajectories return arbitrarily close to themselves infinitely often. We now introduce a complete set of square integrable orthonormal functions $\chi_{\ell}(\boldsymbol{\eta}), l \in \mathbb{Z}$, i.e.,

$$
\sum_{l} \chi_{l}^{*}\left(\boldsymbol{\eta}\left(\mathbf{x}_{0}\right)\right) \chi_{l}(\boldsymbol{\eta}(\mathbf{x}))=\delta\left(\boldsymbol{\eta}\left(\mathbf{x}_{0}\right)-\boldsymbol{\eta}(\mathbf{x})\right)
$$

and

$$
\int d \boldsymbol{\eta} \chi_{l^{\prime}}^{*}(\boldsymbol{\eta}) \chi_{l}(\boldsymbol{\eta})=\delta_{l^{\prime}, l} .
$$

Classical Liouville eigenfunctions for chaotic systems $\rho_{E, \lambda}^{l}(\mathbf{x})$ are then defined as

$$
\rho_{E, \lambda}^{l}(\mathbf{x})=\frac{1}{\sqrt{2 \pi}} \delta(E-H(\mathbf{x})) e^{i \lambda \tau(\mathbf{x})} \chi_{l}(\boldsymbol{\eta}(\mathbf{x})) .
$$

Here the integer $l$ labels the infinite but countable degeneracy of the Liouville eigenvalue $\lambda$ for the continuous part of the spectrum [12] at energy $E$. The distributions $\rho_{E, \lambda}^{l}(\mathbf{x})$ are supported over the entire energy surface $E=H(\mathbf{x})$ and so are in some sense constructed from every orbit of the energy surface. It can be readily verified that these eigenfunctions are both orthogonal and complete (see Appendix A):

$$
\begin{gathered}
\int d \mathbf{x} \rho_{E^{\prime}, \lambda^{\prime}}^{l^{\prime} *}(\mathbf{x}) \rho_{E, \lambda}^{l}(\mathbf{x})=\delta_{l, l^{\prime}} \delta\left(E-E^{\prime}\right) \delta\left(\lambda-\lambda^{\prime}\right), \\
\sum_{l} \int_{0}^{\infty} d E \int_{-\infty}^{\infty} d \lambda \rho_{E, \lambda}^{l *}\left(\mathbf{x}_{0}\right) \rho_{E, \lambda}^{l}(\mathbf{x})=\delta\left(\mathbf{x}-\mathbf{x}_{0}\right)
\end{gathered}
$$

Here we note that the introduction of the complete set of $\chi_{l}$ is necessary to achieve the correct $\delta\left(\mathbf{x}-\mathbf{x}_{\mathbf{0}}\right)$ term in Eq. (38) and that these eigenfunctions are quite different from those proposed elsewhere, which we have shown to be incorrect [17].

The eigenfunctions in Eq. (37) have a structure that at first sight appears to violate several well-known properties of chaotic systems. For example, the existence of stationary square integrable functions $\chi_{l}(\boldsymbol{\eta}(\mathbf{x}))$, which are not functions of the Hamiltonian, appears to contradict the fact that for ergodic systems the only stationary square integrable distributions are functions of the Hamiltonian. However, the $\chi_{l}(\boldsymbol{\eta}(\mathbf{x}))$ distributions are not square integrable on the energy surface. That is,

$$
\int d H d \tau d \boldsymbol{\eta} \delta(E-H)\left|\chi_{l}(\boldsymbol{\eta}(\mathbf{x}))\right|^{2}=\int d \tau=\infty .
$$

In addition, it might appear objectionable that $\chi_{l}(\boldsymbol{\eta}(\mathbf{x}))$ is a global constant of the motion independent of the Hamiltonian. However, this is not the case since the set of points $\mathbf{x}$ satisfying $\chi_{l}(\boldsymbol{\eta}(\mathbf{x}))=\chi^{\prime}$, where $\chi^{\prime}$ is some constant, at most contains points from a countable number of trajectories and so cannot divide the phase space in any meaningful way. Thus the functions $\chi_{l}(\boldsymbol{\eta}(\mathbf{x}))$ are not true constants of the motion.

The relation between these eigenfunctions and the Liouville spectrum on the energy surface $E=H(\mathbf{x})$ is not immediately clear. Examination of the spectral decomposition in Eq. (38) reveals no separation of point and continuous spectrum, although the Liouville eigenfunctions have the correct degeneracy for the continuous spectrum. A proper treatment of the separation of point and continuous spectrum is given in Sec. V B.

\section{CORRESPONDENCE: INTEGRABLE SYSTEMS}

Having established the essential features of the classical eigenstate picture we now examine correspondence. We assume that $r^{\prime}=r$, i.e., that the complete set of commuting quantum observables has as many members as there are constants of the classical motion.

Given the complete set of commuting observables $\hat{H}, \hat{K}_{1}, \ldots, \hat{K}_{r-1}$ for $r \leqslant s$, the complete set of $2 r$ commuting superoperators is given by [Eq. (5)]

$$
\hat{L}, \mathcal{H}, \frac{1}{\hbar}\left[\hat{K}_{1},\right], \frac{1}{2}\left[\hat{K}_{1},\right]_{+}, \ldots, \frac{1}{\hbar}\left[\hat{K}_{r-1},\right], \frac{1}{2}\left[\hat{K}_{r-1},\right]_{+} .
$$


We denote the simultaneous eigenstates of the $r$ observables by $|\mathbf{n}\rangle$ where $\mathbf{n}=\left(n_{1}, \ldots, n_{r}\right)$ are the quantum numbers, and the corresponding eigenvalues are $E_{\mathbf{n}}, K_{1}(\mathbf{n}), \ldots, K_{r-1}(\mathbf{n})$. We further denote the Wigner-Weyl representation of the quantum observables $\hat{H}, \hat{K}_{j}$ by $H(\mathbf{x})$, and $K_{j}^{w}(\mathbf{x})$, for $j=1, \ldots, r-1$.

The Liouville eigenstates $\rho_{\mathbf{n}, \mathbf{m}}^{w}$ must be an eigenstate of a corresponding set of $2 r$ superoperators in the Wigner-Weyl representation, which are

$$
\begin{gathered}
L(\mathbf{x}), \mathcal{H}(\mathbf{x}), \frac{2 i}{\hbar} K_{j}^{w}(\mathbf{x}) \sin \left(\frac{\hbar \sigma}{2}\right), K_{j}^{w}(\mathbf{x}) \cos \left(\frac{\hbar \sigma}{2}\right), \ldots \\
\ldots, \frac{2 i}{\hbar} K_{r-1}^{w}(\mathbf{x}) \sin \left(\frac{\hbar \sigma}{2}\right), K_{r-1}^{w}(\mathbf{x}) \cos \left(\frac{\hbar \sigma}{2}\right),
\end{gathered}
$$

with $\quad L(\mathbf{x})=(2 i / \hbar) H(\mathbf{x}) \sin (\hbar \sigma / 2) \quad$ and $\quad \mathcal{H}(\mathbf{x})$ $=H(\mathbf{x}) \cos (\hbar \sigma / 2)$. By comparison the classical complete set is

$$
L_{c}(\mathbf{x}), H(\mathbf{x}), i\left\{K_{1}(\mathbf{x}),\right\}, K_{1}(\mathbf{x}), \ldots, i\left\{K_{r-1}(\mathbf{x}),\right\}, K_{r-1}(\mathbf{x}) .
$$

We assume that $\lim _{h \rightarrow 0} K_{j}^{w}(\mathbf{x})=K_{j}(x)$. Since $\lim _{h \rightarrow 0} \cos (\hbar \sigma / 2)=1$ and $\lim _{h \rightarrow 0}(2 i / \hbar) \sin (\hbar \sigma / 2)=i \sigma$, we note that formally the quantum operator set [Eq. (41)] becomes the classical operator set [Eq. (42)] in the classical limit. The first eigenequation is the time-independent von Neumann (quantum Liouville) equation

$$
L(\mathbf{x}) \rho_{\mathbf{n}, \mathbf{m}}^{w}(\mathbf{x})=\lambda_{\mathbf{n}, \mathbf{m}} \rho_{\mathbf{n}, \mathbf{m}}^{w}(\mathbf{x}),
$$

where $L(\mathbf{x})$ is the quantum Liouville operator in the WignerWeyl representation and $\lambda_{\mathbf{n}, \mathbf{m}}=\left(E_{\mathbf{n}}-E_{\mathbf{m}}\right) / \hbar$. Second, $\rho_{\mathbf{n}, \mathbf{m}}^{w}$ must satisfy the eigenequation for the Hermitian energy operator $\hat{\mathcal{H}}=\frac{1}{2}[\hat{H},]_{+}$, i.e., the anticommutator equation

$$
\begin{aligned}
\frac{1}{2}\left([\hat{H},|\mathbf{n}\rangle\langle\mathbf{m}|]_{+}\right)^{w} & \equiv H(\mathbf{x}) \cos (\hbar \sigma / 2) \rho_{\mathbf{n}, \mathbf{m}}^{w}(\mathbf{x}) \\
& =\frac{E_{\mathbf{n}}+E_{\mathbf{m}}}{2} \rho_{\mathbf{n}, \mathbf{m}}^{w}(\mathbf{x})
\end{aligned}
$$

or

$$
\mathcal{H}(\mathbf{x}) \rho_{\mathbf{n}, \mathbf{m}}^{w}(\mathbf{x})=E_{\mathbf{n}, \mathbf{m}} \rho_{\mathbf{n}, \mathbf{m}}^{w}(\mathbf{x}),
$$

where $E_{\mathbf{n}, \mathbf{m}}=\left(E_{\mathbf{n}}+E_{\mathbf{m}}\right) / 2$. Similarly, for $j=1, \ldots, r-1$, eigenfunctions $\rho_{\mathbf{n}, \mathbf{m}}^{w}(\mathbf{x})$ satisfy the equations

$$
\begin{aligned}
\left(\frac{1}{\hbar}\left[\hat{K}_{j},|\mathbf{n}\rangle\langle\mathbf{m}|\right]\right)^{w} & \equiv \frac{2 i}{\hbar} K_{j}^{w}(\mathbf{x}) \sin (\hbar \sigma / 2) \rho_{\mathbf{n}, \mathbf{m}}^{w}(\mathbf{x}) \\
& =\frac{K_{j}(\mathbf{n})-K_{j}(\mathbf{m})}{\hbar} \rho_{\mathbf{n}, \mathbf{m}}^{w}(\mathbf{x}),
\end{aligned}
$$

$$
\begin{aligned}
\left(\frac{1}{2}\left[\hat{K}_{j},|\mathbf{n}\rangle\langle\mathbf{m}|\right]_{+}\right)^{w} & \equiv K_{j}^{w}(\mathbf{x}) \cos (\hbar \sigma / 2) \rho_{\mathbf{n}, \mathbf{m}}^{w}(\mathbf{x}) \\
& =\frac{K_{j}(\mathbf{n})+K_{j}(\mathbf{m})}{2} \rho_{\mathbf{n}, \mathbf{m}}^{w}(\mathbf{x}) .
\end{aligned}
$$

We can readily explore the classical limit of the $2 r$ quantum eigenequations, Eqs. (43)-(47), if

$$
\lim _{h \rightarrow 0} \rho_{\mathbf{n}, \mathbf{m}}^{w}(\mathbf{x}) \rightarrow \rho_{\mathbf{n}, \mathbf{m}}^{c}(\mathbf{x}),
$$

where $\rho_{\mathbf{n}, \mathbf{m}}^{c}(\mathbf{x})$ is to be determined. However, this assumption is not generally valid. That is, for quantum systems whose classical analogs are chaotic $\left(r=r^{\prime}=1\right)$, as we demonstrate in Ref. [6], individual quantum eigenfunctions $\rho_{n, m}^{w}(\mathbf{x}), n \neq m$, display essential singularities as $h \rightarrow 0$, and hence do not have correspondence limits. Rather, the spectral projection operators introduced later below (Sec. V) do have correspondence limits. Nonetheless, we can adopt Eq. (48) primarily for notational convenience; the arguments that follow can be reformulated in terms of quantum and classical spectral projection operators when Eq. (48) is invalid.

We assume that the $h \rightarrow 0$ limit of $K_{j}^{w}(\mathbf{x})$ is $K(\mathbf{x})$. In particular, if we consider the case where the set of $2 r$ quantum operators [Eq. (41)] gives the classical set [Eq. (42)] in the classical limit and that Eq. (48) holds then each of the eigenvalue equations [Eqs. (43)-(47)] reduces to the set of classical eigenvalue equations for $\rho_{\lambda, \alpha}(\mathbf{x})$ with the same eigenvalues as in the quantum eigenequations. If this is the case then the quantum $\rho_{\mathbf{n}, \mathbf{m}}^{w}(\mathbf{x})$ goes to the classical $\rho_{\lambda, \alpha}(\mathbf{x})$, with clearly identifiable eigenvalues and correspondence is established.

An example of this approach, applied to integrable systems, is provided below.

\section{A. Integrable systems: Formal correspondence}

In this section we first apply this approach to determine the complete set of correspondence rules for integrable systems. The resulting picture of correspondence for integrable systems is exceptionally clear but gives no insight into the approach to the classical limit. This is treated in the next subsection.

In this instance Eq. (48) has been proven by Berry [15] and by Jaffé and Brumer [10] and is clarified in Sec. IV B below. Thus, in accord with the previous section, the eigenfunctions $\rho_{\mathbf{n}, \mathbf{m}}^{w}(\mathbf{x})$ satisfy $2 s$ classical eigenequations in the classical limit:

$$
\begin{gathered}
L_{c}(\mathbf{x}) \rho_{\mathbf{n}, \mathbf{m}}^{c}(\mathbf{x})=\lambda_{\mathbf{n}, \mathbf{m}} \rho_{\mathbf{n}, \mathbf{m}}^{c}(\mathbf{x}), \\
H(\mathbf{x}) \rho_{\mathbf{n}, \mathbf{m}}^{c}(\mathbf{x})=E_{\mathbf{n}, \mathbf{m}} \rho_{\mathbf{n}, \mathbf{m}}^{c}(\mathbf{x}), \\
i\left\{K_{j}(\mathbf{I}),\right\} \rho_{\mathbf{n}, \mathbf{m}}^{c}(\mathbf{I}, \boldsymbol{\theta})=\frac{K_{j}(\mathbf{n})-K_{j}(\mathbf{m})}{\hbar} \rho_{\mathbf{n}, \mathbf{m}}^{c}(\mathbf{I}, \boldsymbol{\theta}), \\
K_{j}(\mathbf{I}) \rho_{\mathbf{n}, \mathbf{m}}^{c}(\mathbf{I}, \boldsymbol{\theta})=\frac{K_{j}(\mathbf{n})+K_{j}(\mathbf{m})}{2} \rho_{\mathbf{n}, \mathbf{m}}^{c}(\mathbf{I}, \boldsymbol{\theta}),
\end{gathered}
$$


for $j=1, \ldots, s-1$. As in Eq. (28) we conclude that $\rho_{\mathbf{n}, \mathbf{m}}^{c}(\mathbf{x})$ is proportional to

$$
\delta(E-H(\mathbf{x})) \Pi_{j=1}^{s-1} \delta\left(K_{j}(\mathbf{x})-K_{j}^{\prime}\right)
$$

where $E=E_{\mathbf{n}, \mathbf{m}}$, and $K_{j}^{\prime}=\left[K_{j}(\mathbf{n})+K_{j}(\mathbf{m})\right] / 2$. Since $H(\mathbf{x})=H(\mathbf{I})$, and $K_{j}(\mathbf{x})=K_{j}(\mathbf{I})$ for $j=1, \ldots, s-1$ there exists an $\mathbf{I}_{\mathbf{n}, \mathbf{m}}$ such that

$$
\delta(E-H(\mathbf{x})) \Pi_{j=1}^{s-1} \delta\left(K_{j}(\mathbf{x})-K_{j}^{\prime}\right) \propto \delta\left(\mathbf{I}-\mathbf{I}_{\mathbf{n}, \mathbf{m}}\right) .
$$

Hence $\rho_{\mathbf{n}, \mathbf{m}}^{c}(\mathbf{x}) \propto \delta\left(\mathbf{I}-\mathbf{I}_{\mathbf{n}, \mathbf{m}}\right) F_{\mathbf{n}, \mathbf{m}}(\boldsymbol{\theta})$.

From Eqs. (49) and (51) it then follows that

$$
-i \boldsymbol{\omega}\left(\mathbf{I}_{\mathbf{n}, \mathbf{m}}\right) \cdot \frac{\partial}{\partial \boldsymbol{\theta}} F_{\mathbf{n}, \mathbf{m}}(\boldsymbol{\theta})=\frac{E_{\mathbf{n}}-E_{\mathbf{m}}}{\hbar} F_{\mathbf{n}, \mathbf{m}}(\boldsymbol{\theta})
$$

and

$$
-i \frac{\partial K_{j}}{\partial \mathbf{I}}\left(\mathbf{I}_{\mathbf{n}, \mathbf{m}}\right) \cdot \frac{\partial}{\partial \boldsymbol{\theta}} F_{\mathbf{n}, \mathbf{m}}(\boldsymbol{\theta})=\frac{K_{j}(\mathbf{n})-K_{j}(\mathbf{m})}{\hbar} F_{\mathbf{n}, \mathbf{m}}(\boldsymbol{\theta}) .
$$

By WKB $[10,15,16] E_{\mathbf{n}} \sim H\left(\mathbf{I}_{\mathbf{n}}\right)$ where $\mathbf{I}_{\mathbf{n}}=(\mathbf{n}+\boldsymbol{\beta}) \hbar$ and where $\boldsymbol{\beta}$ are the Maslov indices. Similarly we may approximate $K_{j}(\mathbf{n}) \sim K_{j}\left(\mathbf{I}_{\mathbf{n}}\right)$. Assuming that

$$
\mathbf{I}_{\mathbf{n}, \mathbf{m}}-\mathbf{I}_{\mathbf{n}} \sim O(h) \sim \mathbf{I}_{\mathbf{n}, \mathbf{m}}-\mathbf{I}_{\mathbf{m}} \ll \mathbf{I}_{\mathbf{n}, \mathbf{m}}
$$

it follows that

$$
\begin{aligned}
\frac{E_{\mathbf{n}}-E_{\mathbf{m}}}{\hbar} & \sim \frac{H\left(\mathbf{I}_{\mathbf{n}}\right)-H\left(\mathbf{I}_{\mathbf{m}}\right)}{\hbar} \\
& \sim \frac{H\left(\mathbf{I}_{\mathbf{n}, \mathbf{m}}-\left(\mathbf{I}_{\mathbf{n}, \mathbf{m}}-\mathbf{I}_{\mathbf{n}}\right)\right)-H\left(\mathbf{I}_{\mathbf{n}, \mathbf{m}}-\left(\mathbf{I}_{\mathbf{n}, \mathbf{m}}-\mathbf{I}_{\mathbf{m}}\right)\right)}{\hbar} \\
& \sim \boldsymbol{\omega}\left(\mathbf{I}_{\mathbf{n}, \mathbf{m}}\right) \cdot(\mathbf{n}-\mathbf{m})
\end{aligned}
$$

by the Taylor expansion of $H\left(\mathbf{I}_{\mathbf{n}}\right)$ and $H\left(\mathbf{I}_{\mathbf{m}}\right)$ about $\mathbf{I}_{\mathbf{n}, \mathbf{m}}$. Similarly one can show that

$$
\frac{K_{j}(\mathbf{n})-K_{j}(\mathbf{m})}{\hbar} \sim \frac{\partial K_{j}}{\partial \mathbf{I}}\left(\mathbf{I}_{\mathbf{n}, \mathbf{m}}\right) \cdot(\mathbf{n}-\mathbf{m})
$$

Substituting Eqs. (58) and (59) into Eqs. (55) and (56) shows that $F_{\mathbf{n}, \mathbf{m}}(\boldsymbol{\theta}) \sim e^{i(\mathbf{n}-\mathbf{m}) \cdot \boldsymbol{\theta}}$. Thus, up to a normalization factor

$$
\rho_{\mathbf{n}, \mathbf{m}}^{w}(\mathbf{x}) \rightarrow \hbar^{s / 2} \rho_{\mathbf{I}_{\mathbf{n}, \mathbf{m}}, \mathbf{n}-\mathbf{m}}(\mathbf{x})
$$

in the limit as $h \rightarrow 0$.

More specifically, we have to see that $\rho_{\mathbf{n}, \mathbf{m}}^{w}$, an eigenfunction of the $2 s$ quantum operators

$$
\begin{gathered}
L(\mathbf{x}), \mathcal{H}(\mathbf{x}), \frac{2 i}{\hbar} K_{j}^{w}(\mathbf{x}) \sin \left(\frac{\hbar \sigma}{2}\right), K_{j}^{w}(\mathbf{x}) \cos \left(\frac{\hbar \sigma}{2}\right), \ldots \\
\ldots, \frac{2 i}{\hbar} K_{s-1}^{w}(\mathbf{x}) \sin \left(\frac{\hbar \sigma}{2}\right), K_{s-1}^{w}(\mathbf{x}) \cos \left(\frac{\hbar \sigma}{2}\right),
\end{gathered}
$$

with corresponding eigenvalues

$$
\begin{gathered}
\lambda_{\mathbf{n}, \mathbf{m}}, E_{\mathbf{n}, \mathbf{m}}, \frac{K_{1}(\mathbf{n})-K_{1}(\mathbf{m})}{\hbar}, \frac{K_{1}(\mathbf{n})+K_{1}(\mathbf{m})}{2}, \ldots \\
\ldots, \frac{K_{s-1}(\mathbf{n})-K_{s-1}(\mathbf{m})}{\hbar}, \frac{K_{s-1}(\mathbf{n})+K_{s-1}(\mathbf{m})}{2}
\end{gathered}
$$

approaches a classical limit $\rho_{\mathbf{n}, \mathbf{m}}^{c}(\mathbf{I}, \boldsymbol{\theta})=\hbar^{s / 2} \rho_{\mathbf{I}_{\mathbf{n}, \mathbf{m}}, \mathbf{n}-\mathbf{m}}(\mathbf{x})$, which is an eigenfunction of the $2 s$ classical operators

$$
L_{c}(\mathbf{x}), H(\mathbf{x}), i\left\{K_{1}(\mathbf{x}),\right\}, K_{1}(\mathbf{x}), \ldots, i\left\{K_{s-1}(\mathbf{x}),\right\}, K_{s-1}(\mathbf{x}),
$$

with corresponding eigenvalues

$$
\begin{array}{r}
\lambda_{\mathbf{I}_{\mathbf{n}, \mathbf{m}}, \mathbf{n}-\mathbf{m}}, E_{\mathbf{n}, \mathbf{m}},(\mathbf{n}-\mathbf{m}) \cdot \frac{\partial K_{1}}{\partial \mathbf{I}}\left(\mathbf{I}_{\mathbf{n}, \mathbf{m}}\right), \frac{K_{1}(\mathbf{n})+K_{1}(\mathbf{m})}{2}, \\
\ldots,(\mathbf{n}-\mathbf{m}) \cdot \frac{\partial K_{s-1}}{\partial \mathbf{I}}\left(\mathbf{I}_{\mathbf{n}, \mathbf{m}}\right), \frac{K_{s-1}(\mathbf{n})+K_{s-1}(\mathbf{m})}{2} .
\end{array}
$$

Using a completely different argument that employed primitive WKB wave functions Berry showed [15] that the stationary Liouville eigenfunctions have the correspondence limit

$$
\rho_{\mathbf{n}, \mathbf{n}}^{w}(\mathbf{x}) \rightarrow \hbar^{s / 2} \rho_{\mathbf{I}_{\mathbf{n}}, \mathbf{0}}(\mathbf{x}),
$$

where $\mathbf{I}_{\mathbf{n}}=[\mathbf{n}+\boldsymbol{\beta}] \hbar$. Since $\mathbf{I}_{\mathbf{n}, \mathbf{n}} \equiv \mathbf{I}_{\mathbf{n}}$ both results are in agreement. For the case of nonstationary Liouville eigenfunctions Jaffé and Brumer [10] proved the general formula, Eq. (60), where they surmised that $\mathbf{I}_{\mathbf{n}, \mathbf{m}}=\left(\mathbf{I}_{\mathbf{n}}+\mathbf{I}_{\mathbf{m}}\right) / 2$. However, $\mathbf{I}_{\mathbf{n}, \mathbf{m}}$ is obtained correctly by solving the $s$ equations

$$
\left(E_{\mathbf{n}}+E_{\mathbf{m}}\right) / 2=H\left(\mathbf{I}_{\mathbf{n}, \mathbf{m}}\right)
$$

and

$$
\left[K_{j}(\mathbf{n})+K_{j}(\mathbf{m})\right] / 2=K_{j}\left(\mathbf{I}_{\mathbf{n}, \mathbf{m}}\right)
$$

for $j=1, \ldots, s-1$. The surmise of Jaffé and Brumer [10] is an approximation that is good to first order in $h$. To see this note that for $\boldsymbol{\omega}(0) \neq 0$ we can expand $H(\mathbf{I}) \sim \boldsymbol{\omega}(0) \cdot \mathbf{I}+O\left(\mathbf{I}^{2}\right)$. Equation (66) then reduces to $\boldsymbol{\omega}(0) \cdot\left[\mathbf{I}_{\mathbf{n}, \mathbf{m}}-\left(\mathbf{I}_{\mathbf{n}}+\mathbf{I}_{\mathbf{m}}\right) / 2\right]+O\left(h^{2}\right) \sim 0$ for which $\mathbf{I}_{\mathbf{n}, \mathbf{m}} \sim\left(\mathbf{I}_{\mathbf{n}}\right.$ $\left.+\mathbf{I}_{\mathbf{m}}\right) / 2$ is clearly a solution to first order in $h$. If $\partial K_{j}(0) / \partial \mathbf{I} \neq 0$ for all $j$ then it can be shown in a similar fashion that $\mathbf{I}_{\mathbf{n}, \mathbf{m}} \sim\left(\mathbf{I}_{\mathbf{n}}+\mathbf{I}_{\mathbf{m}}\right) / 2$ is a first-order solution of Eqs. (67). Thus, the approximation [10] $\mathbf{I}_{\mathbf{n}, \mathbf{m}} \sim\left(\mathbf{I}_{\mathbf{n}}+\mathbf{I}_{\mathbf{m}}\right) / 2$ is good to first order in Planck's constant.

\section{B. Integrable systems: Approach to the limit}

The method described above assumes the validity of Eq. (48) and fails to provide insights into the behavior of the eigendistributions as $h \rightarrow 0$. Here we extend and reexamine our previous proof [10] of Eq. (48) for integrable systems with particular interest in the mechanism by which essential singularities are avoided as $h \rightarrow 0$. The following argument also determines the correspondence limits of the stationary and nonstationary Liouville eigenfunctions in a unified manner. 
In the $h \rightarrow 0$ limit the eigenfunctions of the Hamiltonian, $\langle\mathbf{q} \mid \mathbf{n}\rangle$, are well approximated by linear combinations of primitive WKB wave functions, i.e.,

$$
\langle\mathbf{q} \mid \mathbf{n}\rangle \sim \sum_{j}(2 \pi)^{-s / 4}\left|\operatorname{det} \frac{\partial^{2} R_{j}\left(\mathbf{q}, \mathbf{I}_{\mathbf{n}}\right)}{\partial \mathbf{q} \partial \mathbf{I}}\right|^{1 / 2} e^{i R_{j}\left(\mathbf{q}, \mathbf{I}_{\mathbf{n}}\right) / \hbar} e^{-i \nu_{j} \pi / 2}
$$

where $R_{j}(\mathbf{q}, \mathbf{I})=\int_{\mathbf{q}_{0}}^{\mathbf{q}} \mathbf{p}_{j}\left(\mathbf{q}^{\prime}, \mathbf{I}\right) \cdot d \mathbf{q}^{\prime}$ are the actions corresponding to the different branches of the multivalued momentum functions $\mathbf{p}_{j}(\mathbf{q}, \mathbf{I})$, and $\nu_{j}$ are the Maslov indices. Thus, it follows that

$$
\begin{aligned}
\rho_{\mathbf{n}, \mathbf{m}}^{w}(\mathbf{x}) & =h^{-s / 2} \int d \mathbf{v} e^{i \mathbf{p} \cdot \mathbf{v} / \hbar}\langle\mathbf{q}-\mathbf{v} / 2 \mid \mathbf{n}\rangle\langle\mathbf{m} \mid \mathbf{q}+\mathbf{v} / 2\rangle \\
& \sim \sum_{j, k} C_{\mathbf{n}, \mathbf{m}}^{j, k}(\mathbf{x}),
\end{aligned}
$$

where

$$
C_{\mathbf{n}, \mathbf{m}}^{j, k}(\mathbf{x})=(2 \pi h)^{-s / 2} \int d \mathbf{v}\left|\operatorname{det} \frac{\partial^{2} R_{j}\left(\mathbf{q}-\mathbf{v} / 2, \mathbf{I}_{\mathbf{n}}\right)}{\partial \mathbf{q} \partial \mathbf{I}} \operatorname{det} \frac{\partial^{2} R_{k}\left(\mathbf{q}+\mathbf{v} / 2, \mathbf{I}_{\mathbf{m}}\right)}{\partial \mathbf{q} \partial \mathbf{I}}\right|^{1 / 2} e^{i \mathbf{p} \cdot \mathbf{v} / \hbar} e^{i R_{j}\left(\mathbf{q}-\mathbf{v} / 2, \mathbf{I}_{\mathbf{n}}\right) / \hbar} e^{-i R_{k}\left(\mathbf{q}+\mathbf{v} / 2, \mathbf{I}_{\mathbf{m}}\right) / \hbar} e^{i\left(\nu_{j}-\nu_{k}\right) \pi / 2},
$$

and where, in principle, we must retain all contributions to the double sum from the different sheets of the phases. However, one of us has argued [10] that the $j \neq k$ terms should make a negligible contribution to $\rho_{\mathbf{n}, \mathbf{m}}^{w}(\mathbf{x})$ due to the rapidly oscillatory character of their integrands. Before examining the $j=k$ terms that yield the classical limit as $h \rightarrow 0$, we briefly demonstrate that the $j \neq k$ terms are indeed small if some averaging over phase space (essentially an energy average) is performed. (We would prefer to carry out an average over wave functions at different energies, corresponding to a restriction to finite time, as in the chaotic case [6], but it is unclear as to how to do this. We expect the phase-space average to achieve a technically similar result.)

Making the change of variable $\mathbf{v} \rightarrow h \mathbf{v}$ Eq. (70) can be written in the form

$$
C_{\mathbf{n}, \mathbf{m}}^{j, k}(\mathbf{x})=\hbar^{s / 2} \int d \mathbf{v}\left|\operatorname{det} \frac{\partial^{2} R_{j}\left(\mathbf{q}-h \mathbf{v} / 2, \mathbf{I}_{\mathbf{n}}\right)}{\partial \mathbf{q} \partial \mathbf{I}} \operatorname{det} \frac{\partial^{2} R_{k}\left(\mathbf{q}+h \mathbf{v} / 2, \mathbf{I}_{\mathbf{m}}\right)}{\partial \mathbf{q} \partial \mathbf{I}}\right|^{1 / 2} e^{2 \pi i \mathbf{p} \cdot \mathbf{v}} e^{i R_{j}\left(\mathbf{q}-h \mathbf{v} / 2, \mathbf{I}_{\mathbf{n}}\right) / \hbar} e^{-i R_{k}\left(\mathbf{q}+h \mathbf{v} / 2, \mathbf{I}_{\mathbf{m}}\right) / \hbar} e^{i\left(\nu_{j}-\nu_{k}\right) \pi / 2}
$$

Note the presence of essential singularities in each of the factors $e^{i R\left(\mathbf{q}-h \mathbf{v} / 2, \mathbf{I}_{\mathbf{n}}\right) / \hbar}$ and $e^{-i R\left(\mathbf{q}+h \mathbf{v} / 2, \mathbf{I}_{\mathbf{m}}\right) / \hbar}$. Expanding the actions $R$ in powers of $h$, we have

$$
R_{j}\left(\mathbf{q}-h \mathbf{v} / 2, \mathbf{I}_{\mathbf{n}}\right) \sim R_{j}\left(\mathbf{q}, \mathbf{I}_{\mathbf{n}}\right)-\frac{h}{2} \mathbf{p}_{j}\left(\mathbf{q}, \mathbf{I}_{\mathbf{n}}\right) \cdot \widetilde{\mathbf{v}}+\frac{h^{2}}{8} \mathbf{v} \cdot \frac{\partial \mathbf{p}_{j}\left(\mathbf{q}, \mathbf{I}_{\mathbf{n}}\right)}{\partial \mathbf{q}} \cdot \widetilde{\mathbf{v}}
$$

and

$$
R_{k}\left(\mathbf{q}+h \mathbf{v} / 2, \mathbf{I}_{\mathbf{m}}\right) \sim R_{k}\left(\mathbf{q}, \mathbf{I}_{\mathbf{m}}\right)+\frac{h}{2} \mathbf{p}_{k}\left(\mathbf{q}, \mathbf{I}_{\mathbf{m}}\right) \cdot \widetilde{\mathbf{v}}+\frac{h^{2}}{8} \mathbf{v} \cdot \frac{\partial \mathbf{p}_{k}\left(\mathbf{q}, \mathbf{I}_{\mathbf{m}}\right)}{\partial \mathbf{q}} \cdot \widetilde{\mathbf{v}}
$$

We also assume that the determinant factors in the integrand are slowly varying, i.e.,

$$
\left|\operatorname{det} \frac{\partial^{2} R_{j}\left(\mathbf{q}-h \mathbf{v} / 2, \mathbf{I}_{\mathbf{n}}\right)}{\partial \mathbf{q} \partial \mathbf{I}} \operatorname{det} \frac{\partial^{2} R_{k}\left(\mathbf{q}+h \mathbf{v} / 2, \mathbf{I}_{\mathbf{m}}\right)}{\partial \mathbf{q} \partial \mathbf{I}}\right|^{1 / 2} \sim\left|\operatorname{det} \frac{\partial^{2} R_{j}\left(\mathbf{q}, \mathbf{I}_{\mathbf{n}}\right)}{\partial \mathbf{q} \partial \mathbf{I}} \operatorname{det} \frac{\partial^{2} R_{k}\left(\mathbf{q}, \mathbf{I}_{\mathbf{m}}\right)}{\partial \mathbf{q} \partial \mathbf{I}}\right|^{1 / 2}
$$

Here the tilde denotes the transpose (a column vector) of the row vector $\mathbf{v}$. Substituting these expressions into Eq. (71) and performing the integral over $\mathbf{v}$ shows that Eq. (71) becomes

$$
\begin{aligned}
C_{\mathbf{n}, \mathbf{m}}^{j, k}(\mathbf{x})= & (2 i / \pi)^{s / 2}\left|\operatorname{det} \frac{\partial^{2} R_{j}\left(\mathbf{q}, \mathbf{I}_{\mathbf{n}}\right)}{\partial \mathbf{q} \partial \mathbf{I}} \operatorname{det} \frac{\partial^{2} R_{k}\left(\mathbf{q}, \mathbf{I}_{\mathbf{m}}\right)}{\partial \mathbf{q} \partial \mathbf{I}}\right|^{1 / 2}\left[\operatorname{det}\left(\frac{\partial \mathbf{p}_{j}\left(\mathbf{q}, \mathbf{I}_{\mathbf{n}}\right)}{\partial \mathbf{q}}-\frac{\partial \mathbf{p}_{k}\left(\mathbf{q}, \mathbf{I}_{\mathbf{m}}\right)}{\partial \mathbf{q}}\right)\right]^{-1 / 2} e^{i\left[R_{j}\left(\mathbf{q}, \mathbf{I}_{\mathbf{n}}\right)-R_{k}\left(\mathbf{q}, \mathbf{I}_{\mathbf{m}}\right)\right] / \hbar} \\
& \times \exp \left\{-2 i\left(\mathbf{p}-\mathbf{p}_{j, k}\right)\left[\frac{\partial \mathbf{p}_{j}\left(\mathbf{q}, \mathbf{I}_{\mathbf{n}}\right)}{\partial \mathbf{q}}-\frac{\partial \mathbf{p}_{k}\left(\mathbf{q}, \mathbf{I}_{\mathbf{m}}\right)}{\partial \mathbf{q}}\right]^{-1}\left(\widetilde{\mathbf{p}-\mathbf{p}_{j, k}}\right) / \hbar\right\} e^{i\left(\nu_{j}-\nu_{k}\right) \pi / 2}
\end{aligned}
$$

where $\mathbf{p}_{j, k}=\left[\mathbf{p}_{j}\left(\mathbf{q}, \mathbf{I}_{\mathbf{n}}\right)+\mathbf{p}_{k}\left(\mathbf{q}, \mathbf{I}_{\mathbf{m}}\right)\right] / 2$. The contributions $C_{\mathbf{n}, \mathbf{m}}^{j, k}(\mathbf{x})$ are therefore highly oscillatory functions of the phase-space variables.

We now smooth Eq. (75) over intervals of length $\Delta p$ about each momentum variable $p_{l}, l=1, \ldots s$. In the stationary phase approximation (for $h \rightarrow 0$ ) 


$$
\begin{aligned}
& (\Delta p)^{-s} \int_{-\Delta p / 2}^{\Delta p / 2} d \mathbf{u}\left[\operatorname{det}\left(\frac{\partial \mathbf{p}_{j}\left(\mathbf{q}, \mathbf{I}_{\mathbf{n}}\right)}{\partial \mathbf{q}}-\frac{\partial \mathbf{p}_{k}\left(\mathbf{q}, \mathbf{I}_{\mathbf{m}}\right)}{\partial \mathbf{q}}\right)\right]^{-1 / 2} \exp \left\{-2 i\left(\mathbf{p}+\mathbf{u}-\mathbf{p}_{j, k}\right)\left[\frac{\partial \mathbf{p}_{j}\left(\mathbf{q}, \mathbf{I}_{\mathbf{n}}\right)}{\partial \mathbf{q}}-\frac{\partial \mathbf{p}_{k}\left(\mathbf{q}, \mathbf{I}_{\mathbf{m}}\right)}{\partial \mathbf{q}}\right]^{-1}\left(\widetilde{\mathbf{p}}+\mathbf{u}-\mathbf{p}_{j, k}\right) / \hbar\right\} \\
& \rightarrow(\pi \hbar / 2 i)^{s / 2} \mathbf{\Omega}_{\Delta p}\left(\mathbf{p}-\mathbf{p}_{j, k}\right),
\end{aligned}
$$

where $\Omega_{\Delta p}(\mathbf{p})=1 /(\Delta p)^{s}$ if $p_{l} \in\left[p_{l}-\Delta p / 2, p_{l}+\Delta p / 2\right]$ for $l=1, \ldots, s$, and is zero otherwise. It follows that smoothing Eq. (75) over a classically small interval in momentum gives

$$
C_{\mathbf{n}, \mathbf{m}}^{j, k}(\mathbf{x}) \sim \hbar^{s / 2}\left|\operatorname{det} \frac{\partial^{2} R_{j}\left(\mathbf{q}, \mathbf{I}_{\mathbf{n}}\right)}{\partial \mathbf{q} \partial \mathbf{I}} \operatorname{det} \frac{\partial^{2} R_{k}\left(\mathbf{q}, \mathbf{I}_{\mathbf{m}}\right)}{\partial \mathbf{q} \partial \mathbf{I}}\right|^{1 / 2} e^{i\left[R_{j}\left(\mathbf{q}, \mathbf{I}_{\mathbf{n}}\right)-R_{k}\left(\mathbf{q}, \mathbf{I}_{\mathbf{m}}\right)\right] / \hbar} \Omega_{\Delta p}\left(\mathbf{p}-\mathbf{p}_{j, k}\right) e^{i\left(\nu_{j}-\nu_{k}\right) \pi / 2}
$$

Further smoothing $C_{\mathbf{n}, \mathbf{m}}^{j, k}(\mathbf{x})$ over intervals of length $\Delta q$ about each coordinate variable $q_{l}, l=1, \ldots s$, one can show that the $C_{\mathbf{n}, \mathbf{m}}^{j, k}(\mathbf{x})$ contribution to $\rho_{\mathbf{n}, \mathbf{m}}^{w}(\mathbf{x})$ is proportional to

$$
\prod_{l=1}^{s} \operatorname{sinc}\left[\left(p_{j}^{l}-p_{k}^{l}\right) \Delta q / 2 \hbar\right]
$$

where $p_{j}^{l}$ is the $l$ th component of $\mathbf{p}_{j}(\mathbf{q}, \mathbf{I})$. Thus, for $j \neq k$ Eq. (78) is $O\left(h^{s}\right)$, while for $j=k$ Eq. (78) is $O(1)$. Thus, we have shown that smoothing over phase space effectively eliminates contributions from the $j \neq k$ terms.

Focusing now on the $j=k$ terms, and dropping the subscript $j$, it follows that the diagonal contributions take the form

$$
C_{\mathbf{n}, \mathbf{m}}^{j, j}(\mathbf{x}) \sim \hbar^{s / 2}\left|\operatorname{det} \frac{\partial^{2} R\left(\mathbf{q}, \mathbf{I}_{\mathbf{n}}\right)}{\partial \mathbf{q} \partial \mathbf{I}} \operatorname{det} \frac{\partial^{2} R\left(\mathbf{q}, \mathbf{I}_{\mathbf{m}}\right)}{\partial \mathbf{q} \partial \mathbf{I}}\right|^{1 / 2} e^{i\left[R\left(\mathbf{q}, \mathbf{I}_{\mathbf{n}}\right)-R\left(\mathbf{q}, \mathbf{I}_{\mathbf{m}}\right)\right] / \hbar} \delta\left(\mathbf{p}-\frac{1}{2}\left[\mathbf{p}\left(\mathbf{q}, \mathbf{I}_{\mathbf{n}}\right)+\mathbf{p}\left(\mathbf{q}, \mathbf{I}_{\mathbf{m}}\right)\right]\right),
$$

where we have replaced $\Omega_{\Delta p}\left\{\mathbf{p}-\frac{1}{2}\left[\mathbf{p}\left(\mathbf{q}, \mathbf{I}_{\mathbf{n}}\right)+\mathbf{p}\left(\mathbf{q}, \mathbf{I}_{\mathbf{m}}\right)\right]\right\}$ by the delta function $\delta\left(\mathbf{p}-\frac{1}{2}\left[\mathbf{p}\left(\mathbf{q}, \mathbf{I}_{\mathbf{n}}\right)+\mathbf{p}\left(\mathbf{q}, \mathbf{I}_{\mathbf{m}}\right)\right]\right)$ since we are free to choose $\Delta p$ small. Assume now that there is an action $\mathbf{I}_{\mathbf{n}, \mathbf{m}}$ such that $\mathbf{p}\left(\mathbf{q}, \mathbf{I}_{\mathbf{n}, \mathbf{m}}\right) \sim \frac{1}{2}\left[\mathbf{p}\left(\mathbf{q}, \mathbf{I}_{\mathbf{n}}\right)+\mathbf{p}\left(\mathbf{q}, \mathbf{I}_{\mathbf{m}}\right)\right]$ (see Ref. [10]), and we also assume that $\mathbf{I}_{\mathbf{n}}, \mathbf{I}_{\mathbf{m}}$, and $\mathbf{I}_{\mathbf{n}, \mathbf{m}}$ satisfy relation (57), then it readily follows that

$$
\frac{R\left(\mathbf{q}, \mathbf{I}_{\mathbf{n}}\right)-R\left(\mathbf{q}, \mathbf{I}_{\mathbf{m}}\right)}{\hbar}=\frac{R\left[\mathbf{q}, \mathbf{I}_{\mathbf{n}, \mathbf{m}}-\left(\mathbf{I}_{\mathbf{n}, \mathbf{m}}-\mathbf{I}_{\mathbf{n}}\right)\right]-R\left[\mathbf{q}, \mathbf{I}_{\mathbf{n}, \mathbf{m}}-\left(\mathbf{I}_{\mathbf{n}, \mathbf{m}}-\mathbf{I}_{\mathbf{m}}\right)\right]}{\hbar} \sim \frac{\partial R\left(\mathbf{q}, \mathbf{I}_{\mathbf{n}, \mathbf{m}}\right)}{\partial \mathbf{I}} \cdot(\mathbf{n}-\mathbf{m})
$$

by Taylor expansion of $R\left(\mathbf{q}, \mathbf{I}_{\mathbf{n}}\right)$ and $R\left(\mathbf{q}, \mathbf{I}_{\mathbf{m}}\right)$ about $\mathbf{I}_{\mathbf{n}, \mathbf{m}}$. Further since $\partial R(\mathbf{q}, \mathbf{I}) / \partial \mathbf{I}=\boldsymbol{\theta}$, we obtain

$$
C_{\mathbf{n}, \mathbf{m}}^{j, j}(\mathbf{x}) \sim \hbar^{s / 2}\left|\operatorname{det} \frac{\partial^{2} R\left(\mathbf{q}, \mathbf{I}_{\mathbf{n}}\right)}{\partial \mathbf{q} \partial \mathbf{I}} \operatorname{det} \frac{\partial^{2} R\left(\mathbf{q}, \mathbf{I}_{\mathbf{m}}\right)}{\partial \mathbf{q} \partial \mathbf{I}}\right|^{1 / 2} \delta\left(\mathbf{p}-\mathbf{p}\left(\mathbf{q}, \mathbf{I}_{\mathbf{n}, \mathbf{m}}\right)\right) e^{i(\mathbf{n}-\mathbf{m}) \cdot \boldsymbol{\theta}(\mathbf{x})} .
$$

Finally, summing over the different sheets $j$ [Eq. (69)], assuming that for small $h$

$$
\left|\operatorname{det} \frac{\partial^{2} R\left(\mathbf{q}, \mathbf{I}_{\mathbf{n}}\right)}{\partial \mathbf{q} \partial \mathbf{I}} \operatorname{det} \frac{\partial^{2} R\left(\mathbf{q}, \mathbf{I}_{\mathbf{m}}\right)}{\partial \mathbf{q} \partial \mathbf{I}}\right|^{1 / 2} \sim\left|\operatorname{det} \frac{\partial^{2} R\left(\mathbf{q}, \mathbf{I}_{\mathbf{n}, \mathbf{m}}\right)}{\partial \mathbf{q} \partial \mathbf{I}}\right|,
$$

and changing variables in the delta function we have

$$
\rho_{\mathbf{n}, \mathbf{m}}^{w}(\mathbf{x}) \sim \hbar^{s / 2} \delta\left(\mathbf{I}(\mathbf{x})-\mathbf{I}_{\mathbf{n}, \mathbf{m}}\right) e^{i(\mathbf{n}-\mathbf{m}) \cdot \boldsymbol{\theta}(\mathbf{x})},
$$

which is the correct correspondence limit. In particular, $\rho_{\mathbf{n}, \mathbf{m}}^{w}$ becomes the classical Liouville eigenfunction [Eq. (30)] with $\mathbf{I}^{\prime}=\mathbf{I}_{\mathbf{n}, \mathbf{m}}$ and $\mathbf{k}=(\mathbf{n}-\mathbf{m})$ in the classical limit. Two things are noteworthy. First, an average over $\Delta p \Delta q$ was necessary to eliminate highly oscillatory terms in the $j \neq k$ contribution to Eq. (69). Second, under these circumstances the essential singularities in the original expression cancel.

\section{CLASSICAL LIOUVILLE SPECTRAL DECOMPOSITION}

Attempts to follow a similar correspondence approach for chaotic systems fail. That is, Eq. (48) is not true insofar as individual eigendistributions $\rho_{n, m}^{w}(\mathbf{x})$, even when suitably averaged, do not have proper classical limits. Examination of our motivating equations (12) and (20) suggests that a oneto-one correspondence of quantum to classical eigenfunctions is not necessary to establish quantum-classical correspondence as $h \rightarrow 0$. Rather, we require a relation between the quantities in brackets in Eqs. (12) and (20) as $h \rightarrow 0$. In this section this idea is quantified, for classical systems, by introducing the classical spectral projectors that we subsequently relate [6], in the $h \rightarrow 0$ limit, to the analogous quantum projectors. In addition, we provide a general approach to computing the classical projectors for arbitrary systems.

The classical projectors will be seen to offer a better method of describing chaotic systems than do the classical Liouville eigenfunctions introduced above [Eq. (37)]. Specifically, they are not based on the arbitrary set $\chi_{l}$, they are completely defined as opposed to the $\rho_{E, \lambda}^{l}(\mathbf{x})$, which are defined to within an overall phase, and they offer a means of separating the spectrum into continuous and discrete components [21].

We also extend the approach and separate the singular spectrum of the periodic orbits from the rest of the continu- 
ous spectrum. The resulting periodic orbit spectral projection operators are used in the companion paper on correspondence in chaotic systems [6].

To develop an energy-frequency spectral decomposition we must isolate the fixed frequency and energy components of an arbitrary time evolving phase-space probability density $\rho(\mathbf{x}, t)$ for a conservative classical Hamiltonian system of $s$ degrees of freedom. Phase-space probability densities $\rho(\mathbf{x}, t)=\rho(\mathbf{X}(\mathbf{x},-t), 0)$ evolve in classical mechanics in accord with the classical Liouville equation, Eq. (13). Consider that

$$
\rho(\mathbf{x}, t)=\int d \mathbf{x}_{0} \rho\left(\mathbf{x}_{0}, 0\right) \delta\left(\mathbf{x}_{0}-\mathbf{X}(\mathbf{x},-t)\right),
$$

where $\delta\left(\mathbf{x}_{0}-\mathbf{X}(\mathbf{x},-t)\right)$ is the kernel of the Liouville propagator, which can be rewritten as

$$
\rho(\mathbf{x}, t)=\int_{-\infty}^{\infty} d t^{\prime} \delta\left(t-t^{\prime}\right) \int d \mathbf{x}_{0} \rho\left(\mathbf{x}_{0}, 0\right) \delta\left(\mathbf{x}_{0}-\mathbf{X}\left(\mathbf{x},-t^{\prime}\right)\right) .
$$

Making the replacement $\delta\left(t-t^{\prime}\right)=(1 / 2 \pi) \int_{-\infty}^{\infty} d \lambda e^{-i \lambda\left(t-t^{\prime}\right)}$ we obtain

$$
\begin{aligned}
\rho(\mathbf{x}, t)= & \int_{-\infty}^{\infty} d t^{\prime} \frac{1}{2 \pi} \int_{-\infty}^{\infty} d \lambda e^{-i \lambda\left(t-t^{\prime}\right)} \\
& \times \int d \mathbf{x}_{0} \rho\left(\mathbf{x}_{0}, 0\right) \delta\left(\mathbf{x}_{0}-\mathbf{X}\left(\mathbf{x},-t^{\prime}\right)\right) .
\end{aligned}
$$

Finally inserting a closure relation for the energy $1=\int_{0}^{\infty} d E \delta(E-H(\mathbf{x}))$ gives

$$
\rho(\mathbf{x}, t)=\int_{0}^{\infty} d E \int_{-\infty}^{\infty} d \lambda e^{-i \lambda t} \int d \mathbf{x}_{0} \rho\left(\mathbf{x}_{0}, 0\right) \Upsilon_{E, \lambda}\left(\mathbf{x} ; \mathbf{x}_{0}\right),
$$

where

$$
\begin{aligned}
Y_{E, \lambda}\left(\mathbf{x} ; \mathbf{x}_{0}\right)= & \frac{1}{2 \pi} \delta\left(E-H\left(\mathbf{x}_{0}\right)\right) \int_{-\infty}^{\infty} d t^{\prime} e^{i \lambda t^{\prime}} \\
& \times \delta\left(\mathbf{x}_{0}-\mathbf{X}\left(\mathbf{x},-t^{\prime}\right)\right) .
\end{aligned}
$$

Equation (87) provides an expansion of $\rho(\mathbf{x}, t)$ in terms of the classical spectral projection operators $Y_{E, \lambda}$, which are expressed, in Eq. (88), in terms of the dynamics. Specifically, $\Upsilon_{E, \lambda}\left(\mathbf{x} ; \mathbf{x}_{0}\right)$ is the Fourier transform of the kernel of the Liouville propagator, restricted to the energy shell. Equation (87) is a restatement of Eq. (20), which emphasizes the classical spectral projection operators as being central to the analysis of the dynamical evolution of distributions $\rho(\mathbf{x}, t)$.

The distribution $\Upsilon_{E, \lambda}\left(\mathbf{x} ; \mathbf{x}_{0}\right)$, as a function of $\mathbf{x}$, is an eigenfunction of the Liouville operator with eigenvalue $\lambda$. To see this consider that propagation with respect to the $\mathbf{x}$ variables gives

$$
\begin{aligned}
e^{-i L_{c} t} \Upsilon_{E, \lambda}\left(\mathbf{x} ; \mathbf{x}_{0}\right)= & \frac{1}{2 \pi} \delta\left(E-H\left(\mathbf{x}_{0}\right)\right) \int_{-\infty}^{\infty} d t^{\prime} e^{i \lambda t^{\prime}} \\
& \times \delta\left(\mathbf{x}_{0}-\mathbf{X}\left(\mathbf{x},-t^{\prime}-t\right)\right) \\
= & e^{-i \lambda t} \Upsilon_{E, \lambda}\left(\mathbf{x} ; \mathbf{x}_{0}\right)
\end{aligned}
$$

where we have made the change of variable $t^{\prime}+t \rightarrow t^{\prime}$. Similarly, with $L_{c}\left(\mathbf{x}_{0}\right)=i\left[\partial H\left(\mathbf{x}_{0}\right) / \partial \mathbf{x}_{0}\right] J \partial / \partial \mathbf{x}_{0}$ operating on the $\mathbf{x}_{0} \quad$ variables, $\quad L_{c} \Upsilon_{E, \lambda}\left(\mathbf{x} ; \mathbf{x}_{0}\right)=-\lambda \boldsymbol{Y}_{E, \lambda}\left(\mathbf{x} ; \mathbf{x}_{0}\right)$. [Here $J=\left(\begin{array}{c}0-I \\ I 0\end{array}\right)$ is the $(2 s \times 2 s)$-dimensional symplectic matrix [25]]. Thus, $Y_{E, \lambda}\left(\mathbf{x} ; \mathbf{x}_{0}\right)$ is an eigenfunction of $L_{c}$ with eigenvalue $\lambda(-\lambda)$ in the $\mathbf{x}\left(\mathbf{x}_{0}\right)$ variables. These properties are consequences of the fact that $\Upsilon_{E, \lambda}\left(\mathbf{x} ; \mathbf{x}_{0}\right)=\Upsilon_{E,-\lambda}\left(\mathbf{x}_{0} ; \mathbf{x}\right)$, which is in turn a consequence of the fact that $L_{c}$ is unitarily equivalent to $-L_{c}$.

The decomposition in Eq. (87) holds for all distributions and all phase-space points $\mathbf{x}$, including periodic orbits. To show this, suppose that $\mathbf{x}$ lies on a periodic orbit. Then by Eq. (88)

$$
\begin{aligned}
\Upsilon_{E, \lambda}\left(\mathbf{x} ; \mathbf{x}_{0}\right) & =\frac{1}{2 \pi} \delta\left(E-H\left(\mathbf{x}_{0}\right)\right) \int_{-\infty}^{\infty} d t^{\prime} e^{i \lambda t^{\prime}} \delta\left(\mathbf{x}_{0}-\mathbf{X}\left(\mathbf{x},-t^{\prime}\right)\right) \\
& =\frac{1}{2 \pi} \delta\left(E-H\left(\mathbf{x}_{0}\right)\right) \sum_{j=-\infty}^{\infty} e^{i j \lambda \tau} \int_{-\tau / 2}^{\tau / 2} d t^{\prime} e^{i \lambda t^{\prime}} \delta\left(\mathbf{x}_{0}-\mathbf{X}\left(\mathbf{x},-t^{\prime}\right)\right) \\
& =\delta\left(E-H\left(\mathbf{x}_{0}\right)\right) \sum_{j=-\infty}^{\infty} \delta(\lambda-2 \pi j / \tau) \frac{1}{\tau} \int_{-\tau / 2}^{\tau / 2} d t^{\prime} e^{2 \pi i j t^{\prime} / \tau} \delta\left(\mathbf{x}_{0}-\mathbf{X}\left(\mathbf{x},-t^{\prime}\right)\right),
\end{aligned}
$$

where $\tau$ is the fundamental period of the orbit. Thus we have

$$
\Upsilon_{E, \lambda}\left(\mathbf{x} ; \mathbf{x}_{0}\right)=\delta\left(E-H\left(\mathbf{x}_{0}\right)\right) \sum_{j=-\infty}^{\infty} \delta(\lambda-2 \pi j / \tau) \frac{1}{\tau} \int_{-\tau / 2}^{\tau / 2} d t^{\prime} e^{2 \pi i j t^{\prime} / \tau} \delta\left(\mathbf{x}_{0}-\mathbf{X}\left(\mathbf{x},-t^{\prime}\right)\right)
$$

on periodic orbits. Now inserting this expression into the right-hand side of Eq. (87) and performing the integrals over $\lambda$ and $E$ gives 


$$
\begin{aligned}
\rho(\mathbf{x}, t) & =\int d \mathbf{x}_{0} \rho\left(\mathbf{x}_{0}, 0\right) \sum_{j=-\infty}^{\infty} \frac{1}{\tau} \int_{-\tau / 2}^{\tau / 2} d t^{\prime} e^{2 \pi i j\left(t^{\prime}-t\right) / \tau} \delta\left(\mathbf{x}_{0}-\mathbf{X}\left(\mathbf{x},-t^{\prime}\right)\right) \\
& =\int d \mathbf{x}_{0} \rho\left(\mathbf{x}_{0}, 0\right) \sum_{j=-\infty}^{\infty} \int_{-\tau / 2}^{\tau / 2} d t^{\prime} \delta\left(t^{\prime}-t-j \tau\right) \delta\left(\mathbf{x}_{0}-\mathbf{X}\left(\mathbf{x},-t^{\prime}\right)\right) \\
& =\int d \mathbf{x}_{0} \rho\left(\mathbf{x}_{0}, 0\right) \delta\left(\mathbf{x}_{0}-\mathbf{X}(\mathbf{x},-t)\right) .
\end{aligned}
$$

Performing the integrals over $d \mathbf{x}_{0}$ gives the probability density on the point $\mathbf{x}$ of the periodic orbit, as required. Thus, the expansion (87) and its associated closure relation hold for points on periodic orbits.

Since Eq. (87) holds for all $\rho$ and all $\mathbf{x}$ it implies the existence of the closure relation

$$
\int_{0}^{\infty} d E \int_{-\infty}^{\infty} d \lambda \Upsilon_{E, \lambda}\left(\mathbf{x} ; \mathbf{x}_{0}\right)=\delta\left(\mathbf{x}_{0}-\mathbf{x}\right)
$$

Two unsatisfactory issues require more careful attention: First, there is no clear separation of the point spectrum from the continuous spectrum [21]. Second, a comparison of Eq. (87) with Eq. (20) suggests that $\Upsilon_{E, \lambda}\left(\mathbf{x}, \mathbf{x}_{0}\right)$ should relate to the Liouville eigenfunctions $\rho_{E, \lambda}^{l}$ as a product of an eigenfunction in $\mathbf{x}_{0}$ times an eigenfunction in $\mathbf{x}$. These issues are addressed below where we treat the integrable and chaotic cases separately.

\section{A. Integrable systems}

To demonstrate the computation of $\boldsymbol{Y}$ consider integrable systems. Here we change to action angle variables $\mathbf{x}=(\mathbf{I}, \boldsymbol{\theta})$ and $\mathbf{x}_{0}=\left(\mathbf{I}_{0}, \boldsymbol{\theta}_{0}\right)$ so that $\Upsilon_{E, \lambda}$ assumes the form

$$
\begin{aligned}
\Upsilon_{E, \lambda}\left(\mathbf{x} ; \mathbf{x}_{0}\right) & =\frac{1}{2 \pi} \delta\left(E-H\left(\mathbf{I}_{0}\right)\right) \int_{-\infty}^{\infty} d t^{\prime} e^{i \lambda t^{\prime}} \delta\left(\mathbf{I}_{0}-\mathbf{I}\right) \delta\left[\left(\boldsymbol{\theta}_{0}-\boldsymbol{\theta}+\boldsymbol{\omega}(\mathbf{I}) t^{\prime}\right) \bmod 2 \pi\right] \\
& =\frac{1}{2 \pi} \delta\left(E-H\left(\mathbf{I}_{0}\right)\right) \int_{-\infty}^{\infty} d t^{\prime} e^{i \lambda t^{\prime}} \delta\left(\mathbf{I}_{0}-\mathbf{I}\right) \frac{1}{(2 \pi)^{s}} \sum_{\mathbf{k} \in \mathbf{Z}^{s}} e^{-i \mathbf{k} \cdot\left[\boldsymbol{\theta}_{0}-\boldsymbol{\theta}+\boldsymbol{\omega}(\mathbf{I}) t^{\prime}\right]},
\end{aligned}
$$

where we have used the identity [26]

$$
\sum_{\mathbf{j} \in \mathbf{Z}^{s}} \delta\left(\boldsymbol{\theta}_{0}-\boldsymbol{\theta}+\boldsymbol{\omega}(\mathbf{I}) t^{\prime}-2 \pi \mathbf{j}\right)=\frac{1}{(2 \pi)^{s}} \sum_{\mathbf{k} \in \mathbf{Z}^{s}} e^{-i \mathbf{k} \cdot\left[\boldsymbol{\theta}_{0}-\boldsymbol{\theta}+\boldsymbol{\omega}(\mathbf{I}) t^{\prime}\right]} .
$$

In terms of the Liouville eigendistributions $\rho_{\mathbf{I}^{\prime}, \mathbf{n}}(\mathbf{I}, \boldsymbol{\theta})[\mathrm{Eq}$. (30)] Eq. (94) becomes

$$
\Upsilon_{E, \lambda}\left(\mathbf{x} ; \mathbf{x}_{0}\right)=\frac{1}{2 \pi} \delta\left(E-H\left(\mathbf{I}_{0}\right)\right) \sum_{\mathbf{n}} \int d \mathbf{I}^{\prime} \delta\left(\lambda-\mathbf{n} \cdot \boldsymbol{\omega}\left(\mathbf{I}^{\prime}\right)\right) \rho_{\mathbf{I}^{\prime}, \mathbf{n}}^{*}\left(\mathbf{I}_{0}, \boldsymbol{\theta}_{0}\right) \rho_{\mathbf{I}^{\prime}, \mathbf{n}}(\mathbf{I}, \boldsymbol{\theta})
$$

Inserting Eq. (96) into Eq. (87) gives the expansion

$$
\rho(\mathbf{x}, t)=\sum_{\mathbf{n}} \int d \mathbf{I}^{\prime} e^{-i \mathbf{n} \cdot \boldsymbol{\omega}\left(\mathbf{I}^{\prime}\right) t} \int d \mathbf{I}_{0} d \boldsymbol{\theta}_{0} \rho\left(\mathbf{I}_{0}, \boldsymbol{\theta}_{0}, 0\right)\left[\rho_{\mathbf{I}^{\prime}, \mathbf{n}}^{*}\left(\mathbf{I}_{0}, \boldsymbol{\theta}_{0}\right) \rho_{\mathbf{I}^{\prime}, \mathbf{n}}(\mathbf{I}, \boldsymbol{\theta})\right] .
$$

This result suggests a natural definition of spectral projection operators for integrable systems, i.e.,

$$
\mathcal{Y}_{\mathbf{I}^{\prime}, \mathbf{n}}\left(\mathbf{x} ; \mathbf{x}_{0}\right)=\rho_{\mathbf{I}^{\prime}, \mathbf{n}}^{*}\left(\mathbf{x}_{0}\right) \rho_{\mathbf{I}^{\prime}, \mathbf{n}}(\mathbf{x}),
$$

which takes the form of a product of Liouville eigenfunctions. These spectral projection operators satisfy the following symmetry, closure and orthonormality relations:

$$
\mathcal{Y}_{\mathbf{I}^{\prime}, \mathbf{n}}\left(\mathbf{x} ; \mathbf{x}_{0}\right)=\mathcal{Y}_{\mathbf{I}^{\prime},-\mathbf{n}}\left(\mathbf{x}_{0} ; \mathbf{x}\right)
$$

$$
\int d \mathbf{I}^{\prime} \mathcal{Y}_{\mathbf{I}^{\prime}, 0}\left(\mathbf{x} ; \mathbf{x}_{0}\right)+\sum_{\mathbf{n} \neq 0} \int d \mathbf{I}^{\prime} \mathcal{Y}_{\mathbf{I}^{\prime}, \mathbf{n}}\left(\mathbf{x} ; \mathbf{x}_{0}\right)=\delta\left(\mathbf{x}-\mathbf{x}_{0}\right),
$$

and

$$
\int d \mathbf{x} \mathcal{Y}_{\mathbf{I}^{\prime \prime}, \mathbf{n}^{\prime}}^{*}\left(\mathbf{x} ; \mathbf{x}_{0}^{\prime}\right) \mathcal{Y}_{\mathbf{I}^{\prime}, \mathbf{n}}\left(\mathbf{x} ; \mathbf{x}_{0}\right)=\delta_{\mathbf{n}, \mathbf{n}^{\prime}} \delta\left(\mathbf{I}^{\prime \prime}-\mathbf{I}^{\prime}\right) \mathcal{Y}_{\mathbf{I}^{\prime}, \mathbf{n}}\left(\mathbf{x}_{0}^{\prime} ; \mathbf{x}_{0}\right)
$$

Further, the decomposition in Eq. (100) clearly displays the separation of point and continuous spectrum. 


\section{B. Chaotic systems}

The time evolution of a phase-space distribution for a chaotic system that is hyperbolic can be decomposed in the following fashion (see Appendix B):

$$
\begin{aligned}
\rho(\mathbf{x}, t)= & \int_{0}^{\infty} d E \int d \mathbf{x}_{0} \rho\left(\mathbf{x}_{0}, 0\right) \Upsilon_{E}\left(\mathbf{x} ; \mathbf{x}_{0}\right) \\
& +\int_{0}^{\infty} d E f d \lambda e^{-i \lambda t} \int d \mathbf{x}_{0} \rho\left(\mathbf{x}_{0}, 0\right) \Upsilon_{E, \lambda}\left(\mathbf{x} ; \mathbf{x}_{0}\right),
\end{aligned}
$$

where $\Upsilon_{E, \lambda}\left(\mathbf{x} ; \mathbf{x}_{0}\right)$ is given by Eq. (88) and

$$
\Upsilon_{E}\left(\mathbf{x} ; \mathbf{x}_{0}\right)=\frac{\delta\left(E-H\left(\mathbf{x}_{0}\right)\right) \delta(E-H(\mathbf{x}))}{\int d \mathbf{x}^{\prime} \delta\left(E-H\left(\mathbf{x}^{\prime}\right)\right)} .
$$

Here $f d \lambda$ denotes the integral over $\lambda$ with the point spectrum at $\lambda=0$ removed, i.e., $f d \lambda=\int_{-\infty}^{\infty} d \lambda P_{E}$, where $P_{E} \rho=\left[\rho-\langle\rho\rangle_{E}\right]$, and

$$
\langle\rho\rangle_{E}=\frac{\int d \mathbf{x} \delta(E-H(\mathbf{x})) \rho(\mathbf{x}, 0)}{\int d \mathbf{x}^{\prime} \delta\left(E-H\left(\mathbf{x}^{\prime}\right)\right)}
$$

is the microcanonical average of $\rho$. The operator $P_{E}$ projects distributions onto the continuous spectrum part of the Hilbert space. Properties such as the system is ergodic $(\lambda=0$ is a nondegenerate point eigenvalue with a corresponding eigenfunction that is uniform over the energy surface), weak mixing (no point eigenvalues other than $\lambda=0$ ), and positive Kolmogorov entropy (i.e., the remainder of the spectrum is continuous) have been incorporated in Eq. (102) in an obvious way. Since the component of $\rho(\mathbf{x})$ with energy $E$ is given by

$$
\int d \mathbf{x}_{0} \rho\left(\mathbf{x}_{0}, 0\right) \Upsilon_{E}\left(\mathbf{x} ; \mathbf{x}_{0}\right)=\langle\rho\rangle_{E} \delta(E-H(\mathbf{x}))
$$

we see that $\Upsilon_{E}$ projects $\rho(\mathbf{x})$ onto the point spectrum part of the Hilbert space. Similarly, $\Upsilon_{E, \lambda}$ projects an arbitrary distribution onto the continuous spectrum part of the Hilbert space associated with a frequency $\lambda$ and energy $E$. We further note that this set of projection operators is complete,

$$
\int_{0}^{\infty} d E \Upsilon_{E}\left(\mathbf{x} ; \mathbf{x}_{0}\right)+\int_{0}^{\infty} d E f d \lambda \Upsilon_{E, \lambda}\left(\mathbf{x} ; \mathbf{x}_{0}\right)=\delta\left(\mathbf{x}-\mathbf{x}_{0}\right)
$$

and orthogonal:

$$
\int d \mathbf{x} \Upsilon_{E^{\prime}}^{*}\left(\mathbf{x} ; \mathbf{x}_{0}^{\prime}\right) \Upsilon_{E}\left(\mathbf{x} ; \mathbf{x}_{0}\right)=\delta\left(E-E^{\prime}\right) \Upsilon_{E}\left(\mathbf{x}_{0}^{\prime} ; \mathbf{x}_{0}\right)
$$

and

$$
\begin{aligned}
& \int d \mathbf{x} \Upsilon_{E^{\prime}, \lambda^{\prime}}^{*}\left(\mathbf{x} ; \mathbf{x}_{0}^{\prime}\right) \Upsilon_{E, \lambda}\left(\mathbf{x} ; \mathbf{x}_{0}\right) \\
& =\delta\left(E-E^{\prime}\right) \delta\left(\lambda-\lambda^{\prime}\right) \Upsilon_{E, \lambda}\left(\mathbf{x}_{0}^{\prime} ; \mathbf{x}_{0}\right)
\end{aligned}
$$

(These relations are proven in Appendix B.) The distributions $Y_{E}$ and $Y_{E, \lambda}$ are thus the kernels of orthogonal stationary and nonstationary spectral projection operators for the classical Liouville spectrum. We have thus achieved, in this approach, the separation of point and continuous spectrum. It remains to examine the relationship of $Y_{E, \lambda}$ to the Liouville eigenfunctions.

To do so insert Eq. (34) into the definition of $Y_{E, \lambda}$ [Eq.(88)] to obtain

$$
\begin{aligned}
\Upsilon_{E, \lambda}\left(\mathbf{x} ; \mathbf{x}_{0}\right)= & \frac{1}{2 \pi} \delta\left(E-H\left(\mathbf{x}_{0}\right)\right) \delta(E-H(\mathbf{x})) \\
& \times e^{-i \lambda\left[\tau\left(\mathbf{x}_{0}\right)-\tau(\mathbf{x})\right]} \delta\left(\boldsymbol{\eta}\left(\mathbf{x}_{0}\right)-\boldsymbol{\eta}(\mathbf{x})\right) .
\end{aligned}
$$

Inserting Eq. (35) in Eq. (109) and rewriting in terms of the definition of the chaotic Liouville eigenfunctions [Eq. (37)] gives

$$
\Upsilon_{E, \lambda}\left(\mathbf{x} ; \mathbf{x}_{0}\right)=\sum_{l} \rho_{E, \lambda}^{l *}\left(\mathbf{x}_{0}\right) \rho_{E, \lambda}^{l}(\mathbf{x})
$$

We also define stationary Liouville eigenfunctions, $\rho_{E}(\mathbf{x})$, via

$$
\rho_{E}(\mathbf{x})=\frac{\delta(E-H(\mathbf{x}))}{\left[\int d \mathbf{x}^{\prime} \delta\left(E-H\left(\mathbf{x}^{\prime}\right)\right)\right]^{1 / 2}}
$$

such that

$$
\Upsilon_{E}\left(\mathbf{x} ; \mathbf{x}_{0}\right)=\rho_{E}^{*}\left(\mathbf{x}_{0}\right) \rho_{E}(\mathbf{x})
$$

The distributions $\rho_{E}(\mathbf{x})$ and $\rho_{E, \lambda}^{l}(\mathbf{x})$ are zero off the energy shell $E=H(\mathbf{x})$. The eigendistributions $\rho_{E}(\mathbf{x})$ are stationary and uniform over the energy shell and belong to the point spectrum. Distributions $\rho_{E, \lambda}^{l}(\mathbf{x})$ are nonuniform and stationary for $\lambda=0$, and nonuniform and nonstationary for $\lambda \neq 0$. The distributions $\rho_{E, \lambda}^{l}(\mathbf{x})$ belong to the continuous spectrum. Finally, note that $\rho_{E}(\mathbf{x})$ and $\rho_{E, \lambda}^{l}(\mathbf{x})$ are supported over the entire $(2 s-1)$-dimensional energy surface $E=H(\mathbf{x})$, in contrast to the distributions $\rho_{\mathbf{I}^{\prime}, \mathbf{k}}(\mathbf{x})$ for integrable systems that are supported only on the $s$-dimensional torus $\mathbf{I}^{\prime}=\mathbf{I}(\mathbf{x})$. In other words, every orbit of the energy surface $E=H(\mathbf{x})$ contributes to the construction of a chaotic eigenfunction.

Comparing

$$
\Upsilon_{E}\left(\mathbf{x} ; \mathbf{x}_{0}\right)=\rho_{E}^{*}\left(\mathbf{x}_{0}\right) \rho_{E}(\mathbf{x})
$$

with

$$
\Upsilon_{E, \lambda}\left(\mathbf{x} ; \mathbf{x}_{0}\right)=\sum_{l} \rho_{E, \lambda}^{l *}\left(\mathbf{x}_{0}\right) \rho_{E, \lambda}^{l}(\mathbf{x})
$$

we see that the stationary spectral projection operators $\Upsilon_{E}$ are simple products of Liouville eigenfunctions while $Y_{E, \lambda}$ 
are composed of a sum of products of Liouville eigenfunctions. This structure, i.e., a sum over products, has important implications for the correspondence of the nonstationary chaotic quantum Liouville eigenfunctions [6]. Hence we shall prove here that $\Upsilon_{E, \lambda}\left(\mathbf{x} ; \mathbf{x}_{0}\right)$ cannot be rewritten as a simple product of some new, as yet undiscovered, eigenfunctions.

Suppose that we can write

$$
\Upsilon_{E, \lambda}\left(\mathbf{x} ; \mathbf{x}_{0}\right)=\Omega_{E, \lambda}^{*}\left(\mathbf{x}_{0}\right) \Omega_{E, \lambda}(\mathbf{x})
$$

for some unknown distributions $\Omega_{E, \lambda}(\mathbf{x})$. Then, in order to satisfy the orthogonality relation [Eq. (108)] we must have

$$
\int d \mathbf{x} \Omega_{E^{\prime}, \lambda^{\prime}}^{*}(\mathbf{x}) \Omega_{E, \lambda}(\mathbf{x})=\delta\left(E-E^{\prime}\right) \delta\left(\lambda-\lambda^{\prime}\right) .
$$

Equations (115) and (116) along with the definition of $\Upsilon_{E, \lambda}$ then imply that

$$
\begin{aligned}
\delta\left(E-E^{\prime}\right) \delta\left(\lambda-\lambda^{\prime}\right) \Omega_{E, \lambda}(\mathbf{x}) \\
\quad=\frac{1}{2 \pi} \delta(E-H(\mathbf{x})) \int_{-\infty}^{\infty} d t^{\prime} e^{i \lambda t^{\prime}} \Omega_{E^{\prime}, \lambda^{\prime}}\left(\mathbf{X}\left(\mathbf{x},-t^{\prime}\right)\right) \\
\quad=\delta(E-H(\mathbf{x})) \delta\left(\lambda-\lambda^{\prime}\right) \Omega_{E^{\prime}, \lambda^{\prime}}(\mathbf{x}) .
\end{aligned}
$$

Integrating over $\lambda^{\prime}$ and $E^{\prime}$ gives

$$
\Omega_{E, \lambda}(\mathbf{x})=\delta(E-H(\mathbf{x})) \int d E^{\prime} \Omega_{E^{\prime}, \lambda}(\mathbf{x}),
$$

which implies that $\Omega_{E, \lambda}(\mathbf{x}) \propto \delta(E-H(\mathbf{x}))$. Note that $\Upsilon_{E, \lambda}\left(\mathbf{x} ; \mathbf{x}_{0}\right)=0$ unless $\mathbf{x}_{0}$ and $\mathbf{x}$ lie on the same trajectory. Thus, if $\Omega_{E, \lambda}(\mathbf{x})=\delta(E-H(\mathbf{x})) N_{\lambda}(\mathbf{x})$ then $N_{\lambda}^{*}\left(\mathbf{x}_{0}\right) N_{\lambda}(\mathbf{x})$ $=0$ unless $\mathbf{x}_{0}$ and $\mathbf{x}$ lie on the same trajectory. However, if $\mathbf{x}_{0}$ and $\mathbf{x}$ lie on the same trajectory then $H(\mathbf{x})=H\left(\mathbf{x}_{0}\right)$ and so

$$
\begin{aligned}
\Omega_{E, \lambda}^{*}\left(\mathbf{x}_{0}\right) \Omega_{E, \lambda}(\mathbf{x}) & =\delta\left(E-H\left(\mathbf{x}_{0}\right)\right) N_{\lambda}^{*}\left(\mathbf{x}_{0}\right) \delta(E-H(\mathbf{x})) N_{\lambda}(\mathbf{x}) \\
& =\delta(E-H(\mathbf{x}))^{2} N_{\lambda}^{*}\left(\mathbf{x}_{0}\right) N_{\lambda}(\mathbf{x})
\end{aligned}
$$

so that $\Omega_{E, \lambda}^{*}\left(\mathbf{x}_{0}\right) \Omega_{E, \lambda}(\mathbf{x})$ would not even be integrable in a delta-function sense. Thus $\Upsilon_{E, \lambda}$ cannot be written as a simple product of Liouville eigenfunctions.

\section{Periodic orbits}

Periodic orbits play an important role in modern theories of quantization in chaotic systems. Given that Eqs. (87) and (102) hold for points on periodic orbits, and given that hyperbolic systems have a countable number of isolated periodic orbits for any given energy, it is also possible to separate the singular spectrum associated with the periodic orbits from the rest of the continuous spectrum. To do this we define periodic orbit distributions [extracted from Eq. (91)]:

$$
\begin{aligned}
\Upsilon_{E, j}^{k}\left(\mathbf{x} ; \mathbf{x}_{0}\right)= & \delta\left(E-H\left(\mathbf{x}_{0}\right)\right) \frac{1}{\tau_{k}} \int_{-\tau_{k} / 2}^{\tau_{k} / 2} d t^{\prime} e^{2 \pi i j t^{\prime} / \tau_{k}} \\
& \times \delta\left(\mathbf{x}^{k}-\mathbf{X}\left(\mathbf{x},-t^{\prime}\right)\right)
\end{aligned}
$$

where $\mathbf{x}^{k}$ is a point on the $k$ th periodic orbit at energy $E$. (Here again $\tau_{k}$ is the fundamental period of orbit $k$.) Note that the distributions $\Upsilon_{E, 0}^{k}$ have the property that

$$
\frac{\int d \mathbf{x} d \mathbf{x}_{0} \Upsilon_{E, 0}^{k}\left(\mathbf{x} ; \mathbf{x}_{0}\right) F\left(\mathbf{x}_{0}\right) G(\mathbf{x})}{\int d \mathbf{x}^{\prime} \delta\left(E-H\left(\mathbf{x}^{\prime}\right)\right)}=\langle F\rangle_{E}\langle G\rangle_{E, 0}^{k},
$$

where $F$ and $G$ are any two observables, $\langle F\rangle_{E}$ denotes a microcanonical average at energy $E$, and $\langle G\rangle_{E, j}^{k} \equiv\left(1 / \tau_{k}\right) \int_{-\tau_{k} / 2}^{\tau_{k} / 2} d t^{\prime} e^{2 \pi i j t^{\prime} / \tau_{k}} G\left(\mathbf{X}\left(\mathbf{x}^{k}, t^{\prime}\right)\right)$ is the $j$ th Fourier component of $G$ on periodic orbit $k$ at energy $E$. The total closure relation now takes the form

$$
\begin{aligned}
& \int d E \Upsilon_{E}\left(\mathbf{x} ; \mathbf{x}_{0}\right)+\int d E \sum_{j}{ }^{\prime} \sum_{\boldsymbol{\eta}_{k} \in \mathcal{P}} \delta_{\boldsymbol{\eta}\left(\mathbf{x}_{0}\right), \boldsymbol{\eta}_{k}} \Upsilon_{E, j}^{k}\left(\mathbf{x} ; \mathbf{x}_{0}\right) \\
&+\int d E f d \lambda \Upsilon_{E, \lambda}\left(\mathbf{x} ; \mathbf{x}_{0}\right)=\delta\left(\mathbf{x}-\mathbf{x}_{0}\right)
\end{aligned}
$$

where $\mathcal{P} \equiv\{\boldsymbol{\eta} \mid \boldsymbol{\eta}$ labels a periodic orbit $\}$ and the $\boldsymbol{\eta}$ variables are those that we introduced earlier, and where $f d \lambda$ now denotes the integral over $\lambda$ with the point and singular spectrum removed, i.e., $f d \lambda=\int d \lambda S_{E} P_{E}$, where

$$
S_{E} \rho(\mathbf{x})=\rho(\mathbf{x})-\sum_{j} \sum_{\boldsymbol{\eta}_{k} \in \mathcal{P}} \delta_{\boldsymbol{\eta}(\mathbf{x}), \boldsymbol{\eta}_{k}}\langle\rho\rangle_{E, j}^{k}
$$

and so

$$
S_{E} P_{E} \rho(\mathbf{x})=\rho(\mathbf{x})-\langle\rho\rangle_{E}-\sum_{j} \sum_{\boldsymbol{\eta}_{k} \in \mathcal{P}} \delta_{\boldsymbol{\eta}(\mathbf{x}), \boldsymbol{\eta}_{k}}\left\langle\left[\rho-\langle\rho\rangle_{E}\right]\right\rangle_{E, j}^{k}
$$

The distributions $\Upsilon_{E, j}^{k}\left(\mathbf{x} ; \mathbf{x}_{0}\right)$ are asymmetric in the $\mathbf{x}, \mathbf{x}_{0}$ variables, and so a Kronecker delta $\delta_{\boldsymbol{\eta}\left(\mathbf{x}_{0}\right), \boldsymbol{\eta}_{k}}$ is introduced as a factor in the singular spectrum term of Eq. (122) in order to restore the proper symmetry with respect to $\mathbf{x}$ and $\mathbf{x}_{0}$. The prime over the sum on $j$ denotes that the point spectrum has been removed, i.e., $\Sigma_{j}^{\prime}=\Sigma_{j} P_{E}$.

One last aspect of definition (120) should be emphasized. For $j=0$ we may write

$$
\begin{aligned}
\Upsilon_{E, 0}^{k}\left(\mathbf{x} ; \mathbf{x}_{0}\right)= & \delta\left(E-H\left(\mathbf{x}_{0}\right)\right) \frac{1}{\tau_{k}} \int_{-\tau_{k} / 2}^{\tau_{k} / 2} d t^{\prime} \delta\left(\tau\left(\mathbf{p}^{k}, \mathbf{q}^{k}\right)\right. \\
& \left.-\tau(\mathbf{p}, \mathbf{q})+t^{\prime}\right) \delta(E-H(\mathbf{x})) \delta\left(\boldsymbol{\eta}_{k}-\boldsymbol{\eta}(\mathbf{x})\right) \\
= & \tau_{k}^{-1} \delta\left(E-H\left(\mathbf{x}_{0}\right)\right) \delta(E-H(\mathbf{x})) \delta\left(\boldsymbol{\eta}_{k}-\boldsymbol{\eta}(\mathbf{x})\right)
\end{aligned}
$$

But local coordinates $\boldsymbol{\xi}_{k}(\mathbf{x})$ of the Poincare surface of section $[20,27]$, transverse to periodic orbit $k$, may be introduced such that 


$$
\delta\left(\boldsymbol{\eta}_{k}-\boldsymbol{\eta}(\mathbf{x})\right)=\delta\left(\xi_{k}(\mathbf{x})\right) .
$$

Note that $\boldsymbol{\eta}_{k}-\boldsymbol{\eta}(\mathbf{x}) \neq \boldsymbol{\xi}_{k}(\mathbf{x})$ since $\boldsymbol{\eta}(\mathbf{x})$ are global $\tau$-independent variables, while $\boldsymbol{\xi}_{k}(\mathbf{x})$ are $\tau$-dependent local variables, i.e., $\boldsymbol{\xi}_{k}\left(H, \tau+\tau_{k}, \boldsymbol{\eta}\right)=M_{k} \boldsymbol{\xi}_{k}(H, \tau, \boldsymbol{\eta})$ where $M_{k}$ is the stability matrix of orbit $k$. However, since

$$
\delta\left(\boldsymbol{\xi}_{k}\left(H, \tau+\tau_{k}, \boldsymbol{\eta}\right)\right)=\frac{1}{\left|\operatorname{det} M_{k}\right|} \delta\left(\boldsymbol{\xi}_{k}(H, \tau, \boldsymbol{\eta})\right)
$$

and $\left|\operatorname{det} M_{k}\right|=1$, it follows that $\delta\left(\boldsymbol{\xi}_{k}(H, \tau, \boldsymbol{\eta})\right)$ is time independent. Thus, the stationary periodic orbit spectral projection operators can be written in the form

$$
\Upsilon_{E, 0}^{k}\left(\mathbf{x} ; \mathbf{x}_{0}\right)=\tau_{k}^{-1} \delta\left(E-H\left(\mathbf{x}_{0}\right)\right) \delta(E-H(\mathbf{x})) \delta\left(\boldsymbol{\xi}_{k}(\mathbf{x})\right) .
$$

This particular form will be of use in the companion paper [6] on correspondence in chaotic systems in which scar contributions are related to these spectral projectors. Indeed, the analysis of the classical dynamics of distributions, contained in this section, will prove central to the analysis of classicalquantum correspondence in chaotic systems, discussed in the following paper [6].

\section{SUMMARY}

In summary, we have constructed a coherent framework for the study of quantum-classical correspondence. We have emphasized the importance of considering, in each mechanics, the entire set of $2 r$ eigenequations in the Liouville picture. We have then shown how this approach, when combined with demonstrations of correspondence for the Liouville eigenfunctions, allows a complete understanding of correspondence for integrable systems. General methods were then discussed for the construction of Liouville spectral decompositions necessary for the study of correspondence in chaotic systems. These methods were then employed for the construction of Liouville eigenfunctions for chaotic systems. Finally, we reviewed the arguments for the correspondence of Liouville eigenfunctions for quantum systems with integrable classical analogs and showed that the primary mechanism of correspondence is the elimination of essential singularities when there is an averaging over a small range $\Delta p \Delta q$.

In the following paper [6] we show that the correspondence problem for systems with chaotic classical analog can be treated by the methods introduced in this paper.

\section{ACKNOWLEDGMENT}

We thank the Natural Sciences and Engineering Research Council of Canada for support of this work.

\section{APPENDIX A}

Here we will prove the orthogonality and completeness relations (38) for the chaotic Liouville eigenfunctions $\rho_{E, \lambda}^{l}$. To prove Eq. (38) consider that

$$
\begin{aligned}
\int d \mathbf{x} \rho_{E^{\prime}, \lambda^{\prime}}^{l^{\prime} *}\left(\mathbf{x}_{0}\right) \rho_{E, \lambda}^{l}(\mathbf{x}) & =\int d H d \tau d \boldsymbol{\eta} \frac{1}{2 \pi} \int_{-\infty}^{\infty} d \tau e^{i\left(\lambda-\lambda^{\prime}\right) \tau} \delta\left(E-E^{\prime}\right) \delta(H-E) \chi_{l^{\prime}}^{*}(\boldsymbol{\eta}) \chi_{l}(\boldsymbol{\eta}) \\
& =\delta_{l^{\prime}, l} \delta\left(E^{\prime}-E\right) \delta\left(\lambda^{\prime}-\lambda\right),
\end{aligned}
$$

where we have used Eq. (36) and the identity $\delta(x)=(1 / 2 \pi) \int_{-\infty}^{\infty} d y e^{i x y}$.

To show completeness note that

$$
\begin{aligned}
\sum_{l} \int_{0}^{\infty} d E \int_{-\infty}^{\infty} d \lambda \rho_{E, \lambda}^{l *}\left(\mathbf{x}^{\prime}\right) \rho_{E, \lambda}^{l}(\mathbf{x}) & =\frac{1}{2 \pi} \sum_{l} \int_{0}^{\infty} d E \int_{-\infty}^{\infty} d \lambda \delta\left(E-H\left(\mathbf{x}^{\prime}\right)\right) \delta(E-H(\mathbf{x})) e^{i \lambda\left[\tau(\mathbf{x})-\tau\left(\mathbf{x}^{\prime}\right)\right]} \chi_{l}^{*}\left(\boldsymbol{\eta}\left(\mathbf{x}^{\prime}\right)\right) \chi_{l}(\boldsymbol{\eta}(\mathbf{x})) \\
& =\delta\left(H(\mathbf{x})-H\left(\mathbf{x}^{\prime}\right)\right) \delta\left(\tau(\mathbf{x})-\tau\left(\mathbf{x}^{\prime}\right)\right) \delta\left(\boldsymbol{\eta}(\mathbf{x})-\boldsymbol{\eta}\left(\mathbf{x}^{\prime}\right)\right)=\delta\left(\mathbf{x}-\mathbf{x}^{\prime}\right)
\end{aligned}
$$

where we used Eq. (35) and the identity $\delta(x)=(1 / 2 \pi) \int_{-\infty}^{\infty} d y e^{i x y}$ to go from the first line to the second, and Eq. (34) to go from the second line to the third.

\section{APPENDIX B}

Here we will prove the decomposition of Eq. (102) and relations (106)-(108). To begin we will prove relation (102). Let $\rho(\mathbf{x}, t)$ be a solution of the classical Liouville equation. The following decomposition can be verified by inspection:

$$
\rho(\mathbf{x}, t)=\int_{0}^{\infty} d E \int d \mathbf{x}_{0} \rho\left(\mathbf{x}_{0}, 0\right) \frac{\delta\left(E-H\left(\mathbf{x}_{0}\right)\right) \delta(E-H(\mathbf{x}))}{\int d \mathbf{x}^{\prime} \delta\left(E-H\left(\mathbf{x}^{\prime}\right)\right)}+\int_{0}^{\infty} d E \rho_{n s}(\mathbf{x}, t) \delta(E-H(\mathbf{x})),
$$


where

$$
\rho_{n s}(\mathbf{x}, t)=\rho(\mathbf{x}, t)-\langle\rho\rangle_{E},
$$

with $\langle\rho\rangle_{E}$ as given in Eq. (104). Furthermore, we may write

$$
\begin{aligned}
\rho_{n s}(\mathbf{x}, t)= & \int d \mathbf{x}_{0} \rho_{n s}\left(\mathbf{x}_{0}, 0\right) \delta\left(\mathbf{x}_{0}-\mathbf{X}(\mathbf{x},-t)\right) \\
= & \int_{-\infty}^{\infty} d t^{\prime} \delta\left(t^{\prime}-t\right) \int d \mathbf{x}_{0} \rho_{n s}\left(\mathbf{x}_{0}, 0\right) \\
& \times \delta\left(\mathbf{x}_{0}-\mathbf{X}\left(\mathbf{x},-t^{\prime}\right)\right) .
\end{aligned}
$$

Substituting $(1 / 2 \pi) \int_{-\infty}^{\infty} d \lambda e^{i \lambda\left(t^{\prime}-t\right)}$ for $\delta\left(t^{\prime}-t\right)$ in Eq. (B3), inserting $1=\int_{0}^{\infty} d E \delta(E-H(\mathbf{x}))$, and then substituting Eq. (B3) into Eq. (B2) and collecting terms, we obtain

$$
\begin{aligned}
\rho(\mathbf{x}, t)= & \int_{0}^{\infty} d E \int d \mathbf{x}_{0} \rho\left(\mathbf{x}_{0}, 0\right) \Upsilon_{E}\left(\mathbf{x} ; \mathbf{x}_{0}\right) \\
& +\int_{0}^{\infty} d E f d \lambda e^{-i \lambda t} \int d \mathbf{x}_{0} \rho\left(\mathbf{x}_{0}, 0\right) \Upsilon_{E, \lambda}\left(\mathbf{x} ; \mathbf{x}_{0}\right),
\end{aligned}
$$

where $f d \lambda \rho=\int d \lambda P_{E} \rho=\int d \lambda\left[\rho-\langle\rho\rangle_{E}\right]$, which proves Eq. (102). Since Eq. (B4) must hold for all $t$ it must also hold for $t=0$. We thus have shown that

$$
\begin{aligned}
\rho(\mathbf{x}, 0)= & \int_{0}^{\infty} d E \int d \mathbf{x}_{0} \rho\left(\mathbf{x}_{0}, 0\right) \Upsilon_{E}\left(\mathbf{x} ; \mathbf{x}_{0}\right) \\
& +\int_{0}^{\infty} d E f d \lambda \int d \mathbf{x}_{0} \rho\left(\mathbf{x}_{0}, 0\right) \Upsilon_{E, \lambda}\left(\mathbf{x} ; \mathbf{x}_{0}\right)
\end{aligned}
$$

and since this must hold for all $\rho$, relation (106) must be true. Here of course we have used definitions (103) and (88).

[1] L.D. Landau and E.M. Lifshitz, Quantum Mechanics, Nonrelativistic Theory (Pergamon, London, 1958); E. Merzbacher, Quantum Mechanics (Wiley, New York, 1961).

[2] J. Ford, in Directions in Chaos, edited by Hao Bai-Lin (World Scientific, Singapore, 1988), Vol 2.

[3] J. Ford, G. Mantica, and G.H. Ristow, Physica D 50, 493 (1991); J. Ford and M. Ilg, Phys. Rev. A 45, 6165 (1992).

[4] M.V. Berry, in Chaos and Quantum Physics, edited by M.J. Giannoni, A. Voros, and J. Zinn-Justin (North-Holland, Amsterdam, 1991).

[5] J. Wilkie and P. Brumer, Phys. Rev. E 49, 1968 (1994).

[6] J. Wilkie and P. Brumer, following paper, Phys. Rev. A 54, 43 (1996).

[7] B.O. Koopman, Proc. Natl. Acad. Sci. U.S.A. 17, 315 (1931).

[8] P.A.M. Dirac, The Principles of Quantum Mechanics (Oxford University Press, Oxford, 1958).

[9] This approach is entirely consistent with Dirac's approach to the reverse problem, i.e., quantization, where the Poisson
The proof of relation (107) is much simpler. Inserting definition (103) into the left-hand side of relation (107) gives

$$
\begin{aligned}
\int d \mathbf{x} & \Upsilon_{E^{\prime}}^{*}\left(\mathbf{x} ; \mathbf{x}_{0}^{\prime}\right) \Upsilon_{E}\left(\mathbf{x} ; \mathbf{x}_{0}\right) \\
& =\int d \mathbf{x} \frac{\delta\left(E^{\prime}-H\left(\mathbf{x}_{0}^{\prime}\right)\right) \delta\left(E^{\prime}-H(\mathbf{x})\right)}{\left[\int d \mathbf{x}^{\prime} \delta\left(E-H\left(\mathbf{x}^{\prime}\right)\right)\right]^{2}} \cdot \delta\left(E-H\left(\mathbf{x}_{0}\right)\right) \\
& \times \delta(E-H(\mathbf{x}))=\delta\left(E-E^{\prime}\right) \\
& \times \frac{\delta\left(E-H\left(\mathbf{x}_{0}^{\prime}\right)\right) \delta\left(E-H\left(\mathbf{x}_{0}\right)\right)}{\int d \mathbf{x}^{\prime} \delta\left(E-H\left(\mathbf{x}^{\prime}\right)\right)} .
\end{aligned}
$$

Simple manipulations and use of definition (103) then give Eq. (107).

Relation (108) can be proven as follows. From definition (88) it follows that

$$
\begin{aligned}
\int d \mathbf{x} & \Upsilon_{E^{\prime}, \lambda^{\prime}}^{*}\left(\mathbf{x} ; \mathbf{x}_{0}^{\prime}\right) \Upsilon_{E, \lambda}\left(\mathbf{x} ; \mathbf{x}_{0}\right) \\
= & \frac{1}{(2 \pi)^{2}} \delta\left(E-E^{\prime}\right) \int d \mathbf{x} \int_{-\infty}^{\infty} d t d t^{\prime} \delta(E-H(\mathbf{x})) e^{-i \lambda t} \\
& \times e^{i \lambda^{\prime} t^{\prime}} \cdot \delta\left(\mathbf{x}_{0}^{\prime}-\mathbf{X}(\mathbf{x},-t)\right) \delta\left(\mathbf{x}_{0}-\mathbf{X}\left(\mathbf{x},-t^{\prime}\right)\right)
\end{aligned}
$$

and this can be rewritten in the form

$$
\begin{aligned}
\int d & \Upsilon_{E^{\prime}, \lambda^{\prime}}^{*}\left(\mathbf{x} ; \mathbf{x}_{0}^{\prime}\right) \Upsilon_{E, \lambda}\left(\mathbf{x} ; \mathbf{x}_{0}\right) \\
= & \frac{1}{(2 \pi)^{2}} \delta\left(E-E^{\prime}\right) \int_{-\infty}^{\infty} d t d t^{\prime} \delta\left(E-H\left(\mathbf{x}_{0}\right)\right) e^{-i \lambda t} \\
& \times e^{i \lambda^{\prime} t^{\prime}} \cdot \delta\left(\mathbf{x}_{0}-\mathbf{X}\left[\mathbf{x}_{0}^{\prime},-\left(t^{\prime}-t\right)\right]\right)
\end{aligned}
$$

and now changing $t^{\prime} \rightarrow t+t^{\prime}$ we readily obtain Eq. (108). bracket is replaced by the commutator.

[10] C. Jaffé and P. Brumer, J. Chem. Phys. 82, 2330 (1985).

[11] C. Jaffé and P. Brumer, J. Phys. Chem. 88, 4829 (1984).

[12] V.I. Arnold and A. Avez, Ergodic Problems of Classical Mechanics (Addison-Wesley, New York, 1989).

[13] S.R. De Groot and L.G. Suttorp, Foundations of Electrodynamics (North-Holland, Amsterdam, 1972).

[14] This requirement is necessary but not sufficient to completely characterize the classical eigenvalue problem, since spectral degeneracies not associated with a classical constant of the motion occur for chaotic systems [12]. See Sec. III B.

[15] M.V. Berry, Philos. Trans. R. Soc. London Ser. A 287, 237 (1977).

[16] I.C. Percival, Adv. Chem. Phys. 36, 1 (1977); J.B. Delos, ibid. 65, 161 (1986).

[17] For an example of the confusion surrounding classical Liouville eigenfunctions for chaotic systems see our comment [J. Wilkie and P. Brumer, Phys. Rev. Lett. (to be published)] on 
the work of T.O. de Carvalho and M.A.M. de Aguiar, Phys. Rev. Lett. 76, 2690 (1996). A related critical comment can be leveled at T.O. de Carvalho and M.A.M. de Aguiar, J. Phys. A 29, 3597 (1996).

[18] M.V. Berry, J. Phys. A 10, 2083 (1977).

[19] A. Voros, in Stochastic Behavior in Classical and Quantum Hamiltonian Systems, edited by G. Casati and J. Ford (Springer, Berlin, 1979).

[20] M.V. Berry, Proc. R. Soc. London Ser. A 423, 219, (1989).

[21] Our use of the terms point and continuous spectrum and their tacit association with $L^{2}$ and singular functions, respectively, is in accord with Dirac's use of improper functions and consistent with current usage in scattering theory.

[22] To see this, consider that $\omega_{1}, \omega_{2}, \ldots, \omega_{s}$ commensurate implies that $\boldsymbol{\omega}=\left[2 \pi / T\left(\mathbf{I}^{\prime}\right)\right] \mathbf{k}\left(\mathbf{I}^{\prime}\right)$ where $\mathbf{k}\left(\mathbf{I}^{\prime}\right) \in \mathbf{Z}^{s}$. Now note that any orbit originating from $\left(\mathbf{I}^{\prime}, \boldsymbol{\theta}^{\prime}\right)$ at $t=0$ passes through the point

$$
\begin{aligned}
\left(\mathbf{I}^{\prime}, \boldsymbol{\theta}^{\prime}\left[T\left(\mathbf{I}^{\prime}\right)\right]\right) & =\left(\mathbf{I}^{\prime},\left[\boldsymbol{\omega} T\left(\mathbf{I}^{\prime}\right)+\boldsymbol{\theta}^{\prime}\right] \bmod 2 \pi\right) \\
& =\left(\mathbf{I}^{\prime},\left[2 \pi \mathbf{k}\left(\mathbf{I}^{\prime}\right)+\boldsymbol{\theta}^{\prime}\right] \bmod 2 \pi\right)=\left(\mathbf{I}^{\prime}, \boldsymbol{\theta}^{\prime}\right)
\end{aligned}
$$

at time $t=T\left(\mathbf{I}^{\prime}\right)$. Thus every orbit is periodic.

[23] Tori for which the frequencies $\omega_{1}, \omega_{2}, \ldots, \omega_{s}$ are incommensurate are constructed from nonperiodic orbits. This can be seen by supposing that a point $\left(\mathbf{I}^{\prime}, \boldsymbol{\theta}^{\prime}\right)$ of the torus exists such that for some time $t=T$,

$$
\boldsymbol{\theta}^{\prime}(T)=\left(\boldsymbol{\omega} T+\boldsymbol{\theta}^{\prime}\right) \bmod 2 \pi=\boldsymbol{\theta}^{\prime},
$$

which implies that there exists an $\mathbf{l} \in \mathbf{Z}^{s}$ such that $T=2 \pi l_{j} / \omega_{j}$ for all nonzero components $\omega_{j}$ of $\boldsymbol{\omega}$. But this implies that $\omega_{1}, \omega_{2}, \ldots, \omega_{s}$ are commensurate, in contradiction with our assumption. Hence all orbits of an incommensurate torus are nonperiodic.

[24] Jaffé and Brumer (Ref. [11]) had previously proposed these as Liouville eigenfunctions for chaotic systems.

[25] H. Goldstein, Classical Mechanics (Addison-Wesley, New York, 1950).

[26] V.S. Vladimirov, Generalized Functions in Mathematical Physics (Mir, Moscow, 1979).

[27] J.H. Hannay and A.M. Ozorio de Almeida, J. Phys. A 17, 3429 (1984). 